\title{
Hollow Fiber and Nanofiber Membranes in Bioartificial Liver and Neuronal Tissue Engineering
}

\author{
Sabrina Morelli Antonella Piscioneri Simona Salerno Loredana De Bartolo \\ Institute on Membrane Technology, National Research Council of Italy, CNR-ITM, Rende, Italy
}

\section{Keywords}

Fiber membranes · Electrospun fibers · Membrane bioreactor $\cdot$ Liver regeneration · Nerve regeneration

\begin{abstract}
To date, the creation of biomimetic devices for the regeneration and repair of injured or diseased tissues and organs remains a crucial challenge in tissue engineering. Membrane technology offers advanced approaches to realize multifunctional tools with permissive environments well-controlled at molecular level for the development of functional tissues and organs. Membranes in fiber configuration with precisely controlled, tunable topography, and physical, biochemical, and mechanical cues, can direct and control the function of different kinds of cells toward the recovery from disorders and injuries. At the same time, fiber tools also provide the potential to model diseases in vitro for investigating specific biological phenomena as well as for drug testing. The purpose of this review is to present an overview of the literature concerning the development of hollow fibers and electrospun fiber membranes used in bioartificial organs, tissue engineered constructs, and in vitro bioreactors. With the aim to highlight the main biomedical applications of fiberbased systems, the first part reviews the fibers for bioartificial
\end{abstract}

karger@karger.com www.karger.com/cto

(C) 2021 S. Karger AG, Basel

Karger" liver and liver tissue engineering with special attention to their multifunctional role in the long-term maintenance of specific liver functions and in driving hepatocyte differentiation. The second part reports the fiber-based systems used for neuronal tissue applications including advanced approaches for the creation of novel nerve conduits and in vitro models of brain tissue. Besides presenting recent advances and achievements, this work also delineates existing limitations and highlights emerging possibilities and future prospects in this field.

(c) 2021 S. Karger AG, Basel

\section{Introduction}

Inspired by nature that during evolution has developed the most efficient structures, scientists created artificial membranes to simulate the natural system. Artificial membranes share functional similarities with the natural membranes, essentially selective transport of molecules, protection from external environment, recognition, and resistance. This bioinspired approach has

\section{A.P. and S.S. contributed equally to this work.}

Correspondence to:

Sabrina Morelli, s.morelli@itm.cnr.it 
been explored to design hierarchically functional structures like in vivo, such as fibers. Tailor-made membranes in hollow fiber (HF) configuration mimic the anatomical vascular system that is designed to provide adequate transport of nutrients to tissues and organs and take away catabolites and wastes. One of the first applications of HF membranes regarded the hemodialysis for the removal of endogenous and exogenous toxins from blood of patients with renal disease replacing the functions of kidney nephrons [De Bartolo et al., 2017]. HF membranes are also employed for the blood oxygenation and removal of $\mathrm{CO}_{2}$ as an artificial lung. Moreover, membranes are used for plasma therapy and leukodepletion from donor blood. In all therapeutic processes, the HFs are designed on the basis of the structural-functional characteristics of the targeted organ that has to be functionally replaced, becoming a keystone in the medical treatments and devices. An interesting exploitation of fiber advantages is related to the field of bioartificial organs and tissue engineering where fibers perform multiple functions aimed at maintaining homeostatic conditions of tissues and organs. For the engineering of bioartificial organs and/or tissues, membranes must create a permissive environment for cell adhesion and growth in a 3D organization [De Bartolo et al., 2001, 2007b; Drioli and De Bartolo, 2006]. In such systems, HF membranes, thanks to their tubular geometry, are able to perfuse cells with nutrients and metabolites protecting them from shear stress. Proliferating cells are usually compartmentalized in the extracapillary or shell compartment and medium flows in the lumen of fibers. Nutrients and metabolites from the lumen diffuse across the membrane wall and reach cells in the shell compartment; vice versa, catabolites and wastes are taken away from the cell compartment. This configuration allows to grow cells at high density, offering high surface area for adhesion-to-volume ratio $\left(30 \mathrm{~cm}^{2} / \mathrm{cm}^{3}\right)$ and minimizing the limitation to mass transfer since each fiber replicates a blood capillary. The structural properties of membranes such as pore size, porosity, and wall thickness together with the physico-chemical characteristics play a crucial role in the mass transfer of molecules that occurs by diffusion and/or convection in response to existing transmembrane concentration or pressure gradients. Additionally, the membranes used in bioartificial devices for the organ replacement therapy perform immunoprotection functions. In this context, the most common suitable HF membranes have a molecular weight cut-off (MWCO) ranging from 70 to $100 \mathrm{kDa}$ to allow the transport of small molecules excluding proteins with high molecular weight such as immunoglobulins and cells, which are responsi- ble for immune response. The mass transfer of molecules in such devices depends on both diffusion/convection and kinetic mechanisms considering that molecules are simultaneously transported and consumed/produced by cells. This is further exacerbated by the wide range of molecular sizes (from small electrolytes to large proteins) and physico-chemical properties (hydrophilic, hydrophobic molecules) of solutes that must be transported across membranes [Legallais et al., 2018].

Membranes acting as an extracellular matrix (ECM) offer a wide surface area for adhesion of cells and provide at micro- and nano-scale the physical, chemical, and mechanical signals necessary for their differentiation. Membrane surface properties can have a direct influence on cell behavior since the topographical, chemical, and mechanical inputs can affect cell spreading and migration. For this reason, membrane surface can be biochemically and topographically modified to offer the tissue specific cues for promoting cell adhesion and tissue regeneration. Moreover, membranes constituted by electrospun nanofibers, mimicking the hierarchical structure of ECM give the opportunity to engineer constructs that can directly influence the behavior and fate of cells. Owing to their ability to match characteristic length scales of natural physiological subcellular structures, nanofiber membranes are widely used as scaffolds to promote the in vitro and in vivo tissue regeneration [Morelli et al., 2015a; Aragón et al., 2018]. They are designed to have a large volume fraction of interconnected pores to facilitate cell migration and the transport of nutrients during tissue regeneration with interconnecting pores. Electrospun fiber membranes can be composed entirely of close-packed random or oriented fibers, which confer a superficial porous structure that can be controlled by modulating the fiber diameter.

A wide array of polymers is used for fiber preparation including both nondegradable (e.g., polyethersulfone PES; polysulfone - PSf; polyacrylonitrile - PAN; modified polyetheretherketone - PEEK-WC; polypropylene - PP; etc.) and degradable materials (e.g., poly-L-lactic acid PLLA; poly lactic-co-glycolic acid - PLGA; polycaprolactone - PCL; polyurethane - PU; etc.) as well as synthetic and natural polymers on the basis of the final application [Salerno et al., 2017]. For the preparation of scaffolds generally either inorganic or organic materials, natural and synthetic, biodegradable polymers and related copolymers, or a combination of both are used, while for bioartificial organs or bioreactors devoted to cell expansion and culture nondegradable polymers, alone or blended, are preferred. Polymer blending is often used to overcome certain limitations of a single material, as poor me- 
chanical properties, inappropriate degradation rate, inadequate hydrophilic/hydrophobic properties, or hampered biocompatibility. Usually, polymers with moderate wettability are employed for the production of HF or nanofiber membranes in contact with cells in order to promote cell adhesion and medium perfusion. Hydrophobic membranes (e.g., PP) are included in some devices as means for exchange of oxygen and carbon dioxide in an attempt to enhance cell oxygenation. HF and nanofiber membranes are produced by several preparation techniques that include electrospinning or dip coating, extrusion or spinning techniques. Wet phase inversion spinning is widely applied for HF preparation due to the control of parameters involved in the preparation process. The membrane properties can be tailored by modulating the different working variables in order to achieve a desired morphology, porosity, pore size, and wall thickness as well as physico-chemical properties.

Electrospinning is the most preferred technique which, utilizing a high applied voltage and a grounded target, allows the preparation of membranes with a micro- or nanofibrous structure and high porosities.

Membrane bioreactors represent a chance for restoring, maintaining, or improving tissue functions or for designing in vitro physiological models (e.g., liver, neurons, skin, etc.). Modeling approaches such as computational tools enable the prediction and subsequent control of a large number of parameters that mutually influence cells and organs improving the understanding and control of fluid dynamics behavior and mass transfer conditions. Today, computational fluid dynamics (CFD) methodologies are employed to describe the fluid mechanics of the bioreactor, therefore, a biohybrid organ can be mathematically modeled increasing its reproducibility and robustness [Curcio et al., 2012]. In this context, engineering approaches such as those related to residence time distribution analysis, if suitably adapted to meet the complexity of biological systems, will significantly increase the efficiency of the bioconstructs. Since HF membrane bioreactors generally consist of a module packed with straight HFs and isolated cells seeded in the extracapillary space, bioreactors are mathematically simplified by Krogh cylinder assumption which was originally introduced to describe oxygen supply from blood vessels to tissues [Krogh, 1919]. Several bioreactors with different configurations have been developed by using fibers packed in parallel or cross-assembled, and various modeling studies have been reported to describe the oxygen supply, metabolic rates, and transport phenomena under different operational conditions [Khakpour et al., 2017].

Fibers for Bioartificial Liver and Neuronal

Tissue Engineering
Here, we review the HF and electrospun fibers used in bioartificial organs, tissue engineered constructs, and in vitro bioreactors focusing on liver and neuronal tissue as examples of the main biomedical applications. We provide an update on the current fiber-based approaches employed for bioartificial liver and liver regeneration, and the recent progresses that have been made in all the aspects of making fiber-based nerve guide conduits (NGCs), including the design, materials, and fabrication techniques (Table 1).

\section{Fibers for Bioartificial Liver and Tissue Engineering}

\section{Hollow Fiber Membrane Systems for Bioartificial \\ Liver}

The use of HF membranes is widely recognized as feasible and attractive approach in the realization of functional engineered liver tissues and organs for medical, clinical, and preclinical practices, and for advanced research therapies and technologies. HF membranes represent a keystone in the design of bioartificial livers (BALs) to be used in clinical assist therapy as temporary extracorporeal supports, for patients awaiting orthotopic liver transplantation, or allowing the native or post-transplant liver to regenerate and recover. Polymeric membranes offer a 3D niche for liver tissue formation under dynamic and perfused condition, serving likewise as predictable in vitro models to be applied for assessing effects and toxicity of new drugs and therapeutic compounds alternatively or complementary to animal experimentation [Memoli et al., 2010]. Moreover, HF membranes made of biodegradable polymers act as promising materials for producing in vivo implantable liver tissues that undergo to a controlled, predictable, and non-toxic biodegradation and resorption without any surgical removal for the fully integration of the implanted cells into the host.

Polymeric membranes are notably suitable as supporting material in liver tissue engineering thanks to their intrinsic characteristics of efficiency and operational simplicity, high permeability and selectivity to molecular components, excellent stability under mild operational conditions, robust automation and easy scale-up. Furthermore, synthetic membranes can easily be mass produced, modulating their structural and physico-chemical properties for specific applications.

In the first bioartificial liver systems, HF membranes were used as selective barriers allowing solute exchange and the selective mass transport of nutrients and metabolites, and in the meantime the compartmentalization of 


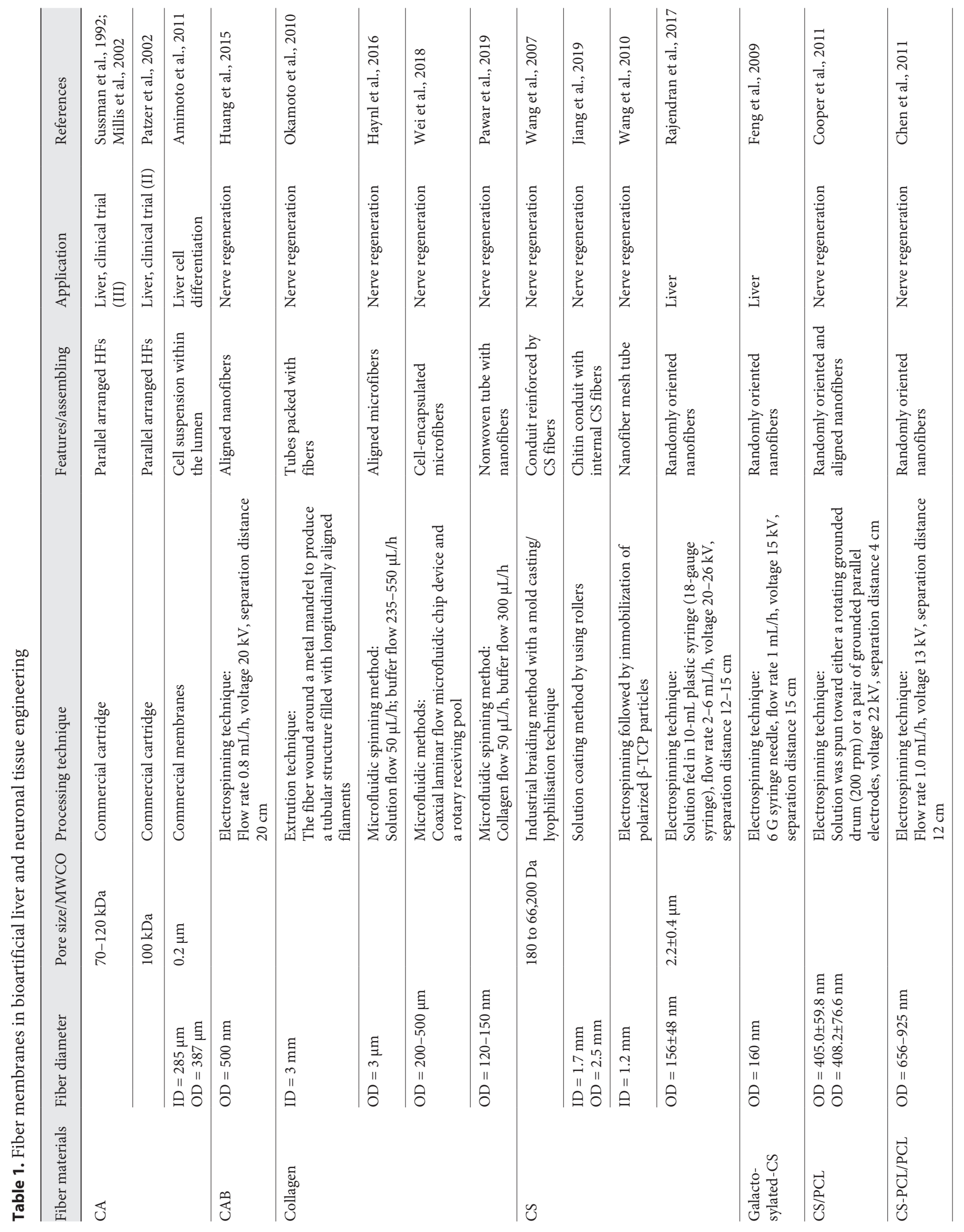




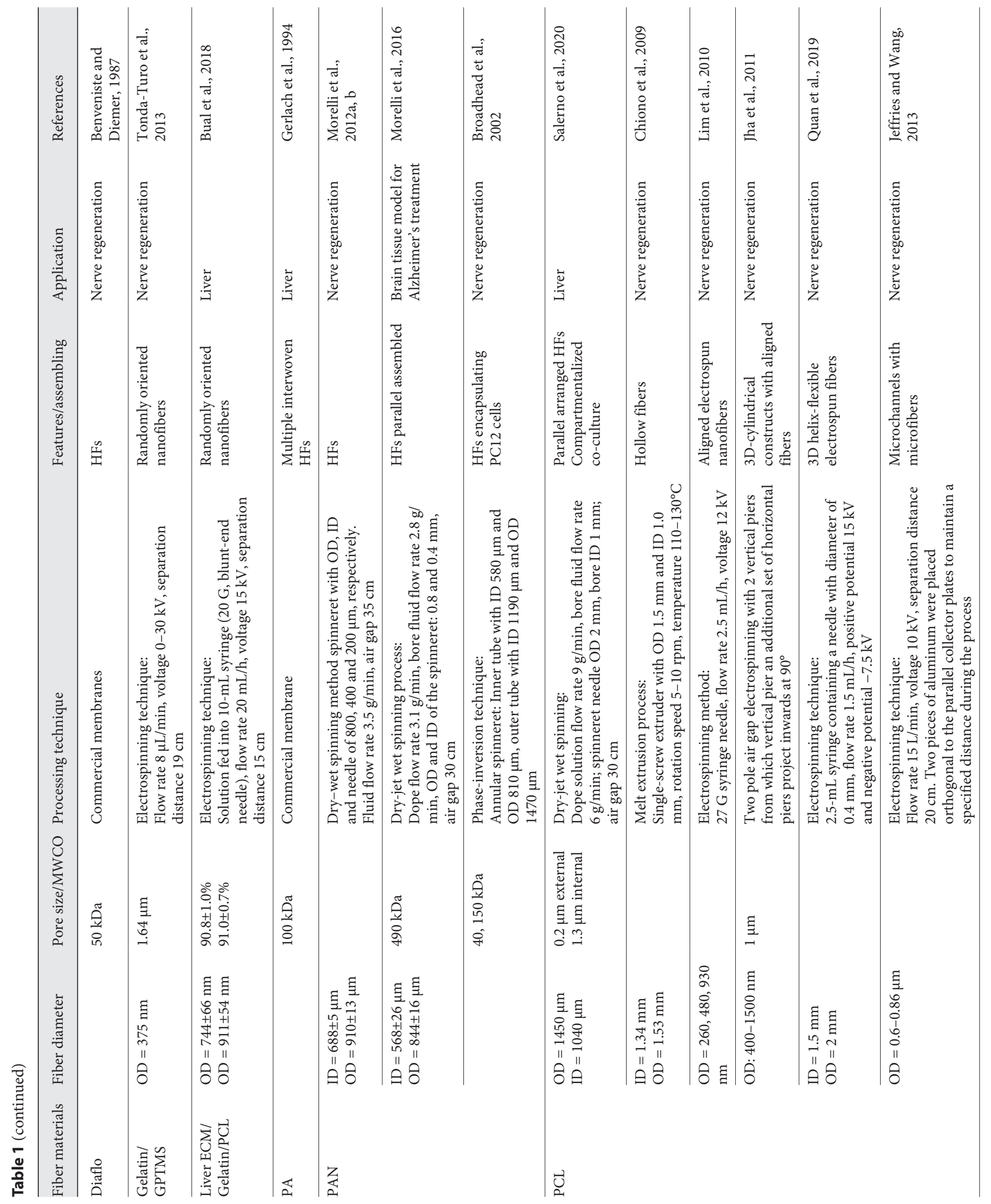




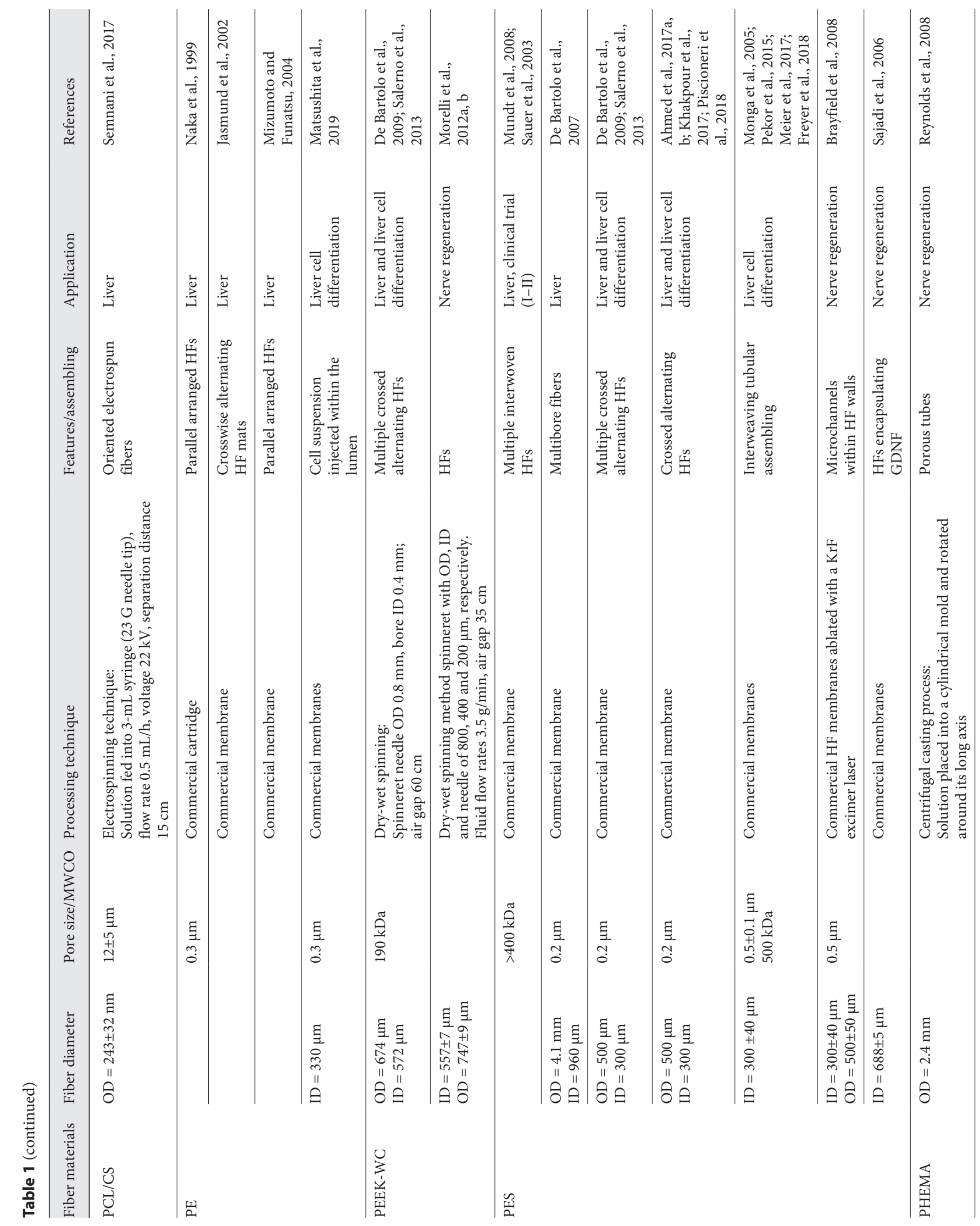




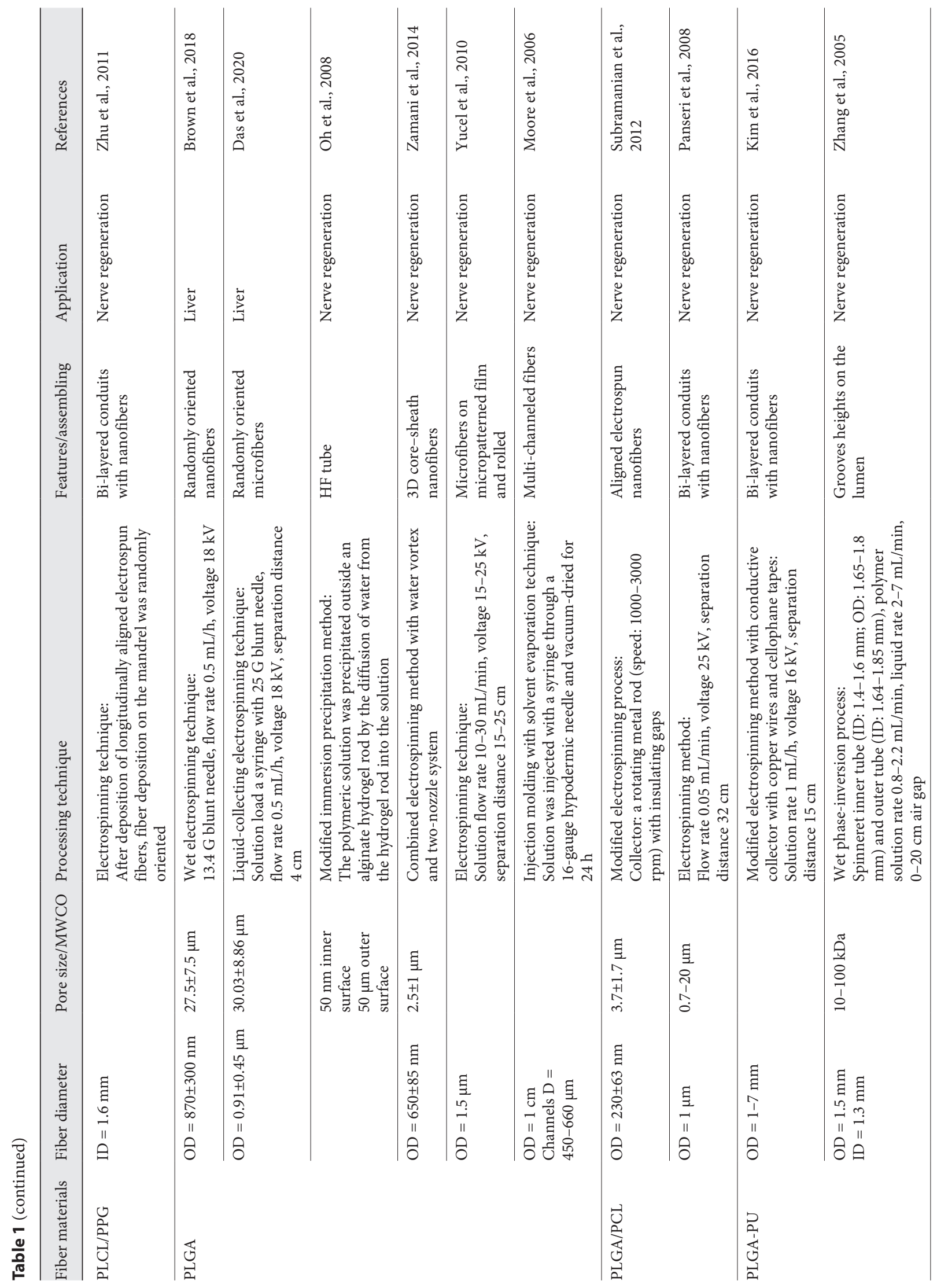




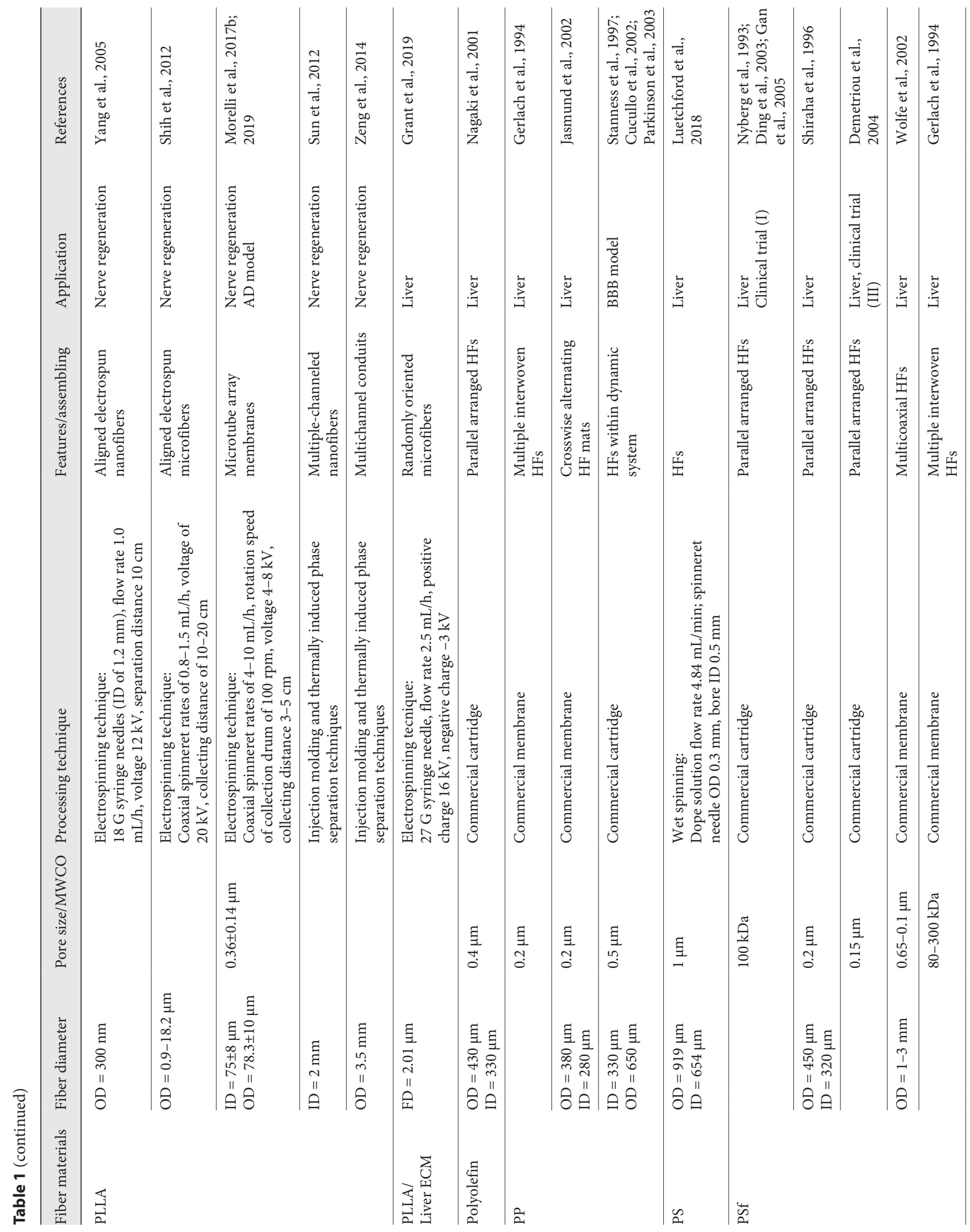




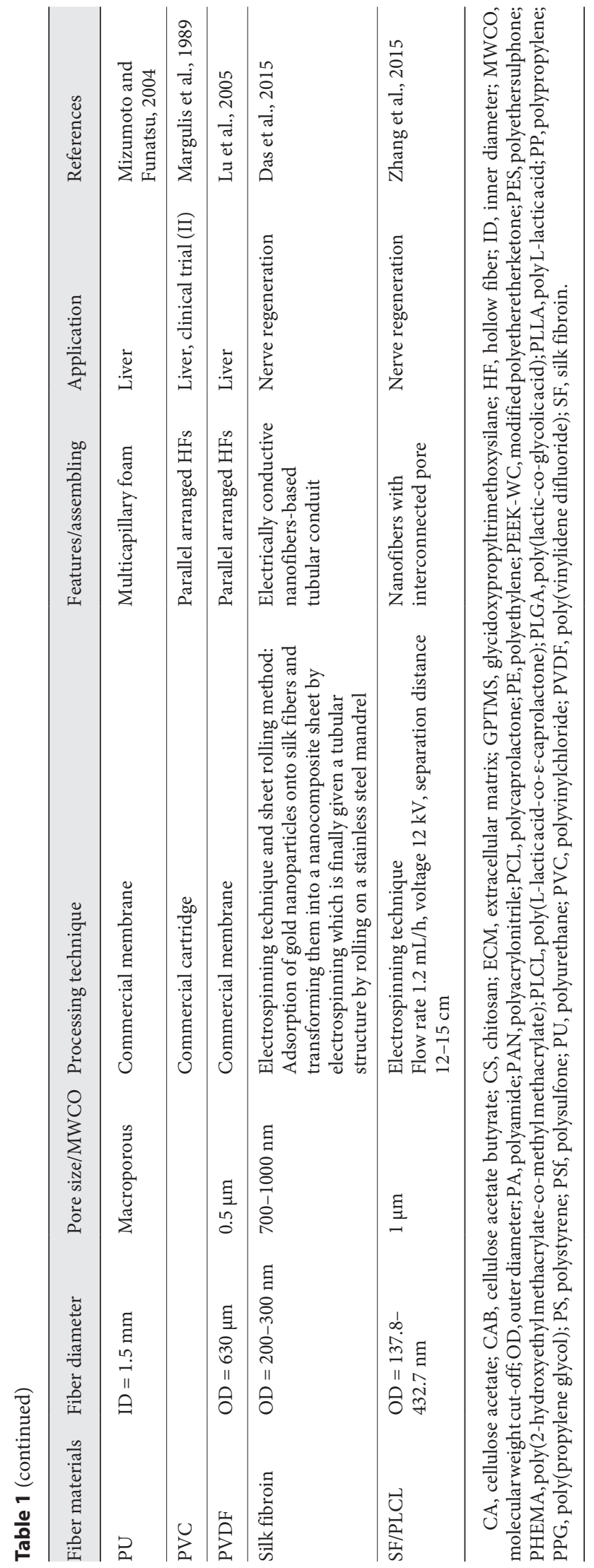

Fibers for Bioartificial Liver and Neuronal Tissue Engineering cells (Fig. 1). Acting as selective barriers, membranes preserve hepatocytes from shear stress due to the perfusion, and ensure their immune-isolation avoiding adverse immune reaction by the patients' hosting cells. Successively, considering that hepatocytes are anchorage-dependent cells, membranes were selected in order to perform a dual task: as supporting scaffold for cell adhesion, mimicking the natural ECM, which is important for the polarization of hepatocytes and organization in a 3-dimensional architecture, and as selective barrier, ensuring the transport of metabolites, nutrients, and specific products to and from cells. Several papers have demonstrated that membranes offer specific cues to hepatocytes for recapitulating the in vivo milieu of the natural microenvironments, driving and supporting 3-dimensional tissue formation as well as ECM protein deposition [De Bartolo et al., 2006; Memoli et al., 2007; Morelli et al., 2007; Piscioneri et al., 2011; Salerno et al., 2011, 2017, 2018; Salerno and De Bartolo, 2017].

Most bioartificial livers were realized by parallel assembling HF membranes for the compartmentalization of hepatocytes in the shell of the fibers. One of the first bioartificial livers that was involved in phase I and II of the first larger clinical trial loaded hepatocytes in free suspension in the extracapillary space of a polyvinylchloride (PVC) HF membrane cartridge [Margulis et al., 1989]. The function and viability of these BALs was limited to few hours, considering that hepatocytes are adherent cells. To improve cell viability, other bioartificial livers maintained hepatocytes in the extracapillary space of parallel assembled HFs, encapsulated in agarose microdroplets [Shiraha et al., 1996], in adhesion to microcarriers [Demetriou et al,. 1995], entrapped in collagen gel [Naka et al., 1999] or in basement membrane matrix [Nagaki et al., 2001].

Few bioreactors compartmentalized liver cells in the lumen of parallel assembled fibers. In this device xenogeneic hepatocytes were entrapped in collagen gel in the lumen of PSf HF membranes with MWCO of $100 \mathrm{kDa}$, and the patient's blood continuously recirculated in the extracapillary space. The membrane cut-off excluded the patient's immunoglobulin and complement proteins from the intraluminal space, thus providing immunoprotection for the xenogeneic hepatocytes [Nyberg et al., 1993].

Several bioartificial livers in clinical trials employed PSf HF membranes (Table 1). The hepatocytes encapsulated in agarose microdroplets were maintained in the extracapillary space of PSf HFs with $0.2 \mu \mathrm{m}$ pore size [Shiraha et al., 1996]. The TECA-Hybrid Artificial Liver Support System (TECA-HALSS) that employed HF cartridges from 


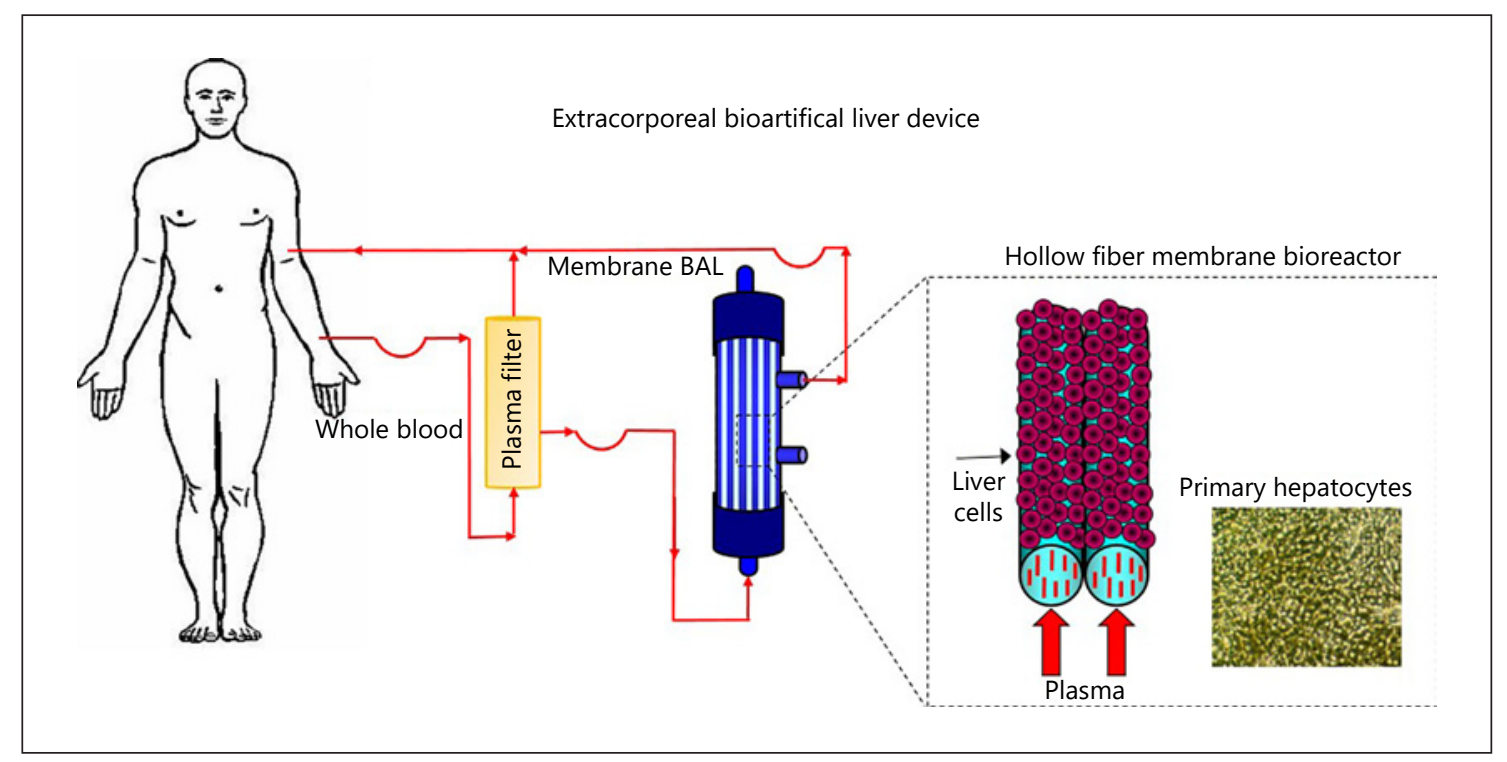

Fig. 1. Scheme of bioartificial liver (BAL) device.

TECA Corporation, and that was tested in phase I clinical trials, was constituted of PSf HFs with $100 \mathrm{kDa}$ cut-off, loaded with hepatocyte spheroids in its extra-fiber space [Ding et al., 2003; Gan et al., 2005]. PSf fibers of $0.15 \mu \mathrm{m}$ pore size were employed in HepatAssist ${ }^{\mathrm{TM}}$ from Circe Biomedical that was tested on a large scale in a phase III clinical trial, and that utilized hepatocytes attached to collagen-coated dextran microcarriers into the extracapillary space. The membrane pore size prevented hepatocytes and cell debris from passing through the fiber wall and entering the patient's bloodstream that flows through the lumen of the fibers [Demetriou et al., 1995, 2004].

Another BAL that underwent extensive phase III clinical trials, the Extra-corporeal Liver Assist Device (ELAD, Vital Therapies, Inc.) consists of 4 cellulose acetate (CA) HF membrane cartridges with a cut-off of $70 \mathrm{kDa}$, that separates $200 \mathrm{~g}$ of hepatoblastoma cell line in the extracapillary space from the patient's plasma flowing in membrane lumens [Sussman et al., 1992]. Successively, the device was improved by increasing the CA membrane cutoff (up to $120 \mathrm{kDa}$ ) together with the cell mass (up to 400 $\mathrm{g}$ for each cartridge), enhancing the oxygenation, and adding filters for preventing cancer cell migration risks [Millis et al., 2002].

HF cartridges of CA membranes with a nominal 100$\mathrm{kDa}$ diffusive size cut-off constitute the bioreactor of the Excorp Medical bioartificial liver support system (BLSS), developed at the University of Pittsburg and involved in phase II trials. In this system, 70-120 g of hepatocytes were embedded in a collagen matrix in the extracapillary space. In contrast to other devices which perfuse patient plasma through the bioreactor, the BLSS perfused whole blood through the bioreactor, with significant advantages in affecting the patient outcome [Patzer et al., 2002].

With the purpose of increasing surface adhesion and paracrine cell communication, a peculiar configuration was realized in a membrane bioreactor in which primary human hepatocytes were compartmentalized in the lumen of modified PES multibore fibers. The fibers consisted of 7 capillaries ( $960 \mu \mathrm{m}$ internal diameter) clustered in a single fiber ( $4.1 \mathrm{~mm}$ outer diameter) through a foamy porous $(0.2 \mu \mathrm{m}$ pore size $)$ and highly permeable support structure, that conferred high stability and mechanical resistance to the hepatic tissue. Cells were cultured in the lumen of different capillaries that were in the same time in communication with each other thanks to the porous wall of the multibore fibers, and the diffusion of their secreted paracrine factors. The surface wettability and the high permeability allowed high adhesion and perfusion of primary human hepatocytes, and the device was used as in vitro model to investigate the liver detoxification of drugs and therapeutic molecules [De Bartolo et al., 2007a].

A similar configuration was realized by culturing hepatocytes in a multicapillary PU foam, consisting of a cylindrical block having many capillaries with internal diameter of $1.5 \mathrm{~mm}$ and forming a flow channel for the culture medium or plasma. In contrast to the previous multibore system, hepatocytes were inoculated in the mi- 
croporous structure of the PU foam between the capillaries and formed spontaneously spheroids of 100-150 $\mu \mathrm{m}$ diameter [Mizumoto and Funatsu, 2004]. The same authors developed a liver lobule-like structure consisting of polyethylene (PE) HFs coated with ethylene vinyl alcohol, regularly arranged close together, among which hepatocytes were inoculated by using a centrifugal force, forming organoids [Mizumoto and Funatsu, 2004].

Different approaches adopted the membrane functionalization with the aim of improving the cell-material interactions. Some of these modifications employ the inclusion of biomolecules on the membrane surface which are commonly recognized by hepatocyte receptors. A poly(vinylidene difluoride) HF surface was modified with the immobilization of a galactosylated polymer [Lu et al., 2005]. PES HF membranes were functionally coated with a glutaraldehyde-crosslinked gelatin that provided an ECM-like environment for hepatocyte cell lines [Verma et al., 2018a]. Other modifications aimed to change physico-chemical membrane surface properties (i.e., polarity and wettability) to enhance the cell adhesion and functions [Salerno et al., 2009] or to improve the performance of liver assist bioreactors. A porous polystyrene HF membrane, developed by using microcrystalline sodium chloride as porogen, was subjected to an oxygen plasma surface treatment to reduce its hydrophobicity [Luetchford et al., 2018]. The biocompatibility and separation performance of PES HF membranes were improved by the incorporation of graphene oxide nanosheets decorated with hydrophilic zeolitic imidazole framework [Modi et al., 2018]. Phospholipid polymers, immobilized by a chemical condensation reaction on both the inner and outer surfaces of $\mathrm{CA} /$ phosphorylcholine co-polymer blended HF membranes, improved the water and solute permeability of membranes, as well as the functionality of hepatocytes [Ho Ye et al., 2006].

Considering that oxygen represents one of the most important limiting nutrients in membrane bioartificial liver, owing to its relatively low solubility and the high uptake rate of liver cells for systems with precarious vascularization, many efforts have been focused on the development of membrane devices with improved oxygenation. To this purpose, different systems included specific HFs devoted to the direct oxygenation of hepatocytes. An oxygenating HF bioreactor (OXY-HFB) was developed by alternating crosswise 2 mats of HF membranes with a constant distance of $200 \mu \mathrm{m}$ porous PP oxygenating HF membranes $(0.2 \mu \mathrm{m}$ pore size $)$ and $\mathrm{PE}$ heat exchange HF membranes. Oxygen requirements and temperature control were supplied through the fibers among

Fibers for Bioartificial Liver and Neuronal

Tissue Engineering which were primary porcine hepatocytes cultured at high cell density in direct contact with the perfused medium [Jasmund et al., 2002].

A more complex HF-based system was realized on the concept device developed by Gerlach et al. [1994], in which hepatocytes were cultured on the outer surface and between 4 independent interwoven capillary membranes, creating a network with a counter-current medium perfusion and internal oxygenation, using polyamide for medium/plasma inflow, PSf for medium/plasma outflow, hydrophobic PP for oxygen/carbon dioxide exchanges, hydrophilic PP for sinusoidal endothelial co-culture. This concept was applied in the Liver Support System (Charitè - Humboldt University, Germany) involved in a phase I clinical trial. An upgrade of the concept co-cultured the endothelial cells in direct contact with the hepatocytes in the extracapillary space created by 2 separate bundles of PES HF membranes with MWCO $>400 \mathrm{kDa}$ for the counter-current medium perfusion, and multilaminate HF membranes for low gradient gas exchanges. The device underwent phase I/II clinical trials [Mundt et al., 2008]. Successively, the interwoven HF bioreactor was integrated into a modular extracorporeal liver support system, and combined with DetoxModule for albumin dialysis [Sauer and Gerlach, 2002], and underwent phase I clinical trials loaded with human hepatocytes [Sauer et al., 2003]. Notwithstanding first encouraging results reporting on 8 patients successfully bridged to liver transplantation, modular extracorporeal liver support never progressed in controlled, randomized clinical trial required for regulatory approval. The miniaturized cell bioreactor, utilizing PES HF membranes with MWCO 400-500 kDa, was used as in vitro model to test liver detoxification of different drugs and their specific biotransformation pathway [Mueller et al., 2011; Hoffmann et al., 2012].

Liver acinar structure and physiological parameters were mimicked in a multicoaxial HF bioreactor, consisting of 4 concentric tubes with increasing diameter placed one inside the other. Hepatocytes were cultured under radial flow in a sandwich configuration in the space between the 2 innermost semi-permeable tubes, consisting of PSf HFs with $1 \mathrm{~mm}$ outer diameter (OD) $/ 0.65 \mu \mathrm{m}$ pore size, and $3 \mathrm{~mm} \mathrm{OD} / 0.1 \mu \mathrm{m}$ pore size, respectively. Integral oxygenation occurred in the outermost compartment created by a third coaxial gas permeable silastic tube and the polycarbonate housing [Wolfe et al., 2002].

Another configuration based on the use of different fibers, was realized by cross assembling in alternating manner 2 bundles of HFs with specific physico-chemical, morphological, and transport properties for medium in- 


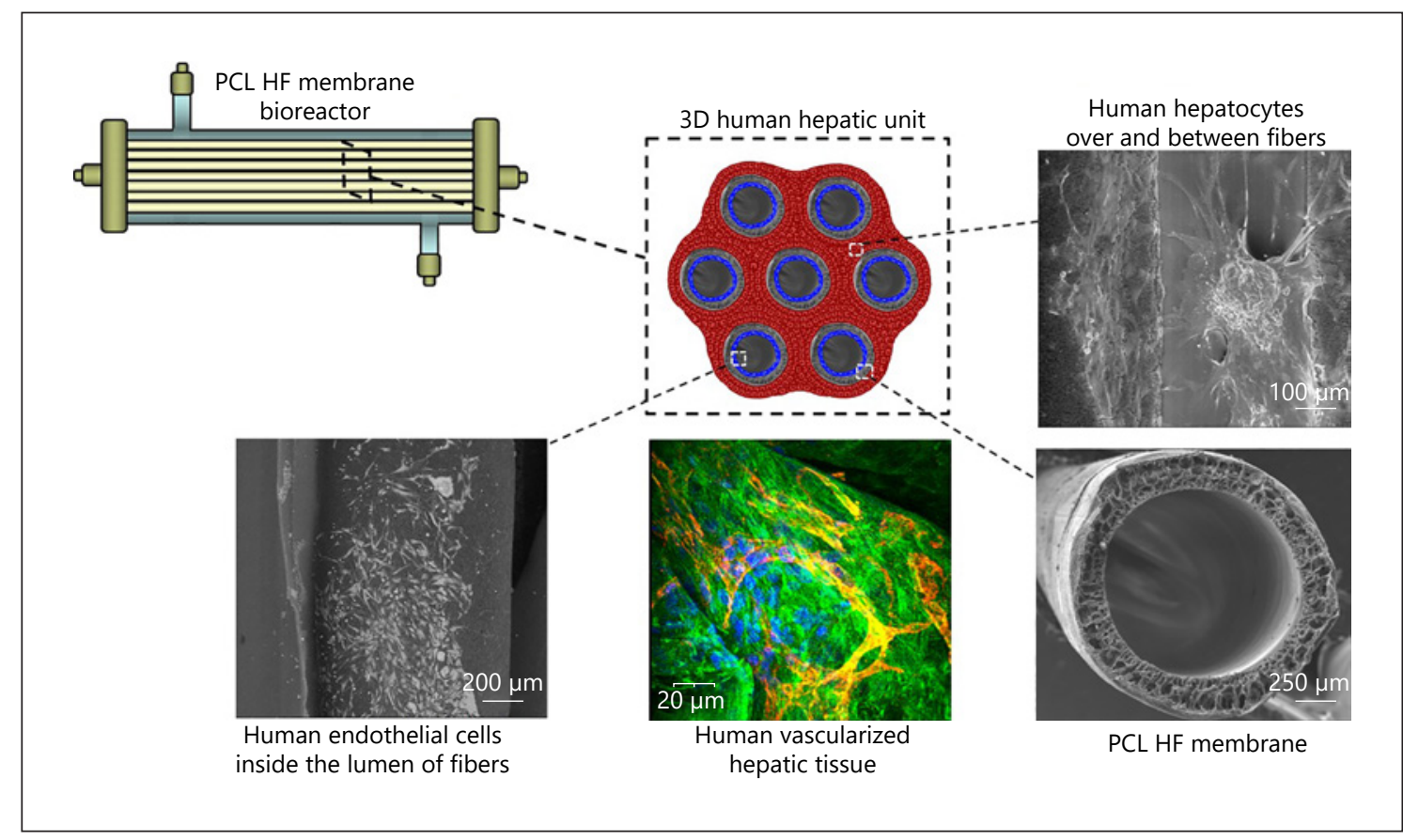

Fig. 2. Human vascularized hepatic tissue realized by using poly( $\varepsilon$-caprolactone) (PCL) hollow fiber (HF) membrane bioreactor. Reprinted and modified from Salerno et al. [2020] (licensed under CC BY 4.0).

flow and outflow, respectively, and creating an extracapillary 3-dimensional network for cell adhesion and culture, with high mass exchange and oxygenation through the cross-flow of the medium. A crossed HF membrane bioreactor was realized by assembling $2 \mathrm{HF}$ bundles of PEEK modified with phthalide cardo group [Zhang et al., 1987] (obtained by solution polycondensation of phenolphthalein and dichlorobenzophenone; PEEK-WC) with a MWCO of $190 \mathrm{kDa}$, devoted to the feeding of oxygenated medium, and PES with $0.2 \mu \mathrm{m}$ of pore size and high hydraulic permeance $\left(J=15.2 \mathrm{~L} / \mathrm{m}^{2} \mathrm{~h} \cdot \mathrm{mbar}\right)$, devoted to the removal of waste medium. The distance among the fibers was set on the basis of the modeled mass transport of nutrients and cell products. The optimized fluid dynamic ensured well mixing microenvironment in which primary human hepatocytes retained their differentiated morpho-functional behavior up to 19 days of culture [De Bartolo et al., 2009]. In another crossed membrane bioreactor, constituted of 2 bundles of only PES HF membranes, a $3 \mathrm{D}$ liver system was realized in which primary human sinusoidal endothelial cells, stellate cells, and hepatocytes were seeded sequentially in order to mimic the in vivo layers of cells. Cells self-assembled, forming tissue-like structures around and between the fibers, with hepatocytes forming aggregates, promoted by stellate cells, and surrounded by sinusoidal tube-like structures [Ahmed et al., 2017a]. Within the bioreactor, a proper oxygenation and supply of nutrients was provided to the cells ensuring a physiological amount that supported moreover the formation and long-term maintenance of functional human liver microtissues. Indeed, in the same bioreactor, human liver microtissue spheroids with uniform size and shape, and formed through self-assembly, were cultured, giving rise to larger microstructures around the fibers and forming liver-like tissue, which retained functional features up to 25 days [Ahmed et al., 2017b; Khakpour et al., 2017].

An innovative strategy was implemented with the aim to realize a vascularized liver tissue by creating vascular channels of endothelial cells that grows among a human liver tissue. To this purpose, biodegradable polycaprolactone HF membranes were synthetized by dry-jet wet phase inversion spinning method, tailoring operational parameters to obtain microporous interconnected pores along the membrane walls, and pores with mean diameter of 0.4 and $1.3 \mu \mathrm{m}$, on the external and internal membrane surface, respectively. A 3D compartmentalized co-culture was realized by loading primary human endothelial cells in the lumen of PCL HF membranes, and primary human hepatocytes in adhesion over and between the fibers, in the extralumen space (Fig. 2). Endothelial cells in 
a such configuration colonized the lumen of the PCL HFs forming vascular channels, and communicated with hepatocytes through their secreted factors that permeated across the microporous membrane walls. The developed PCL HF membranes together with fluid dynamic condition, created a physiologically relevant microenvironment with a large integration of the cells that retained functional activity and drug biotransformation up to 18 days, establishing a bottom-up tissue engineering approach to create $3 \mathrm{D}$ vascularized liver tissues [Salerno et al., 2020].

\section{Hollow Fibers to Induce Hepatic Differentiation}

The efficacy of fiber membrane cell culture models for pharmacological and toxicological research is widely demonstrated. However, the development and validation of a functional liver tissue construct requires a large number of cells (high density culture) and experiments. This point arises one of the major concerns in the field of liver tissue engineering, which is related to the optimal cell source. Primary human hepatocytes still represent the gold standard for hepatic culture models but their scarcity engenders the need to find alternative hepatic cell sources for a consistent production of healthy functional hepatocytes. An attracting possibility for fiber-based liver tools is the use of progenitor/fetal liver cells and/or adult and pluripotent stem cells thanks to their intrinsic proliferative features and their ability to differentiate into hepatocytelike cells [Zeilinger et al., 2016; Hosseini et al., 2019]. Although their use holds promising perspectives, the achievement of standardized differentiation methods is still a huge challenge. To face this unsolved issue, many attempts have been implemented within the scientific community by using different approaches. The various investigated methodologies share the common feature of acting toward the improvement of cell culture environment with the aim to enhance the differentiation potential. In this context, the use of fiber membranes satisfies different criteria reproducing a biomimetic environment that mimics their native niche. Within a fiber membrane system, it is possible to conjugate a proper chemical microenvironment with a proper spatial and temporal exposure to fundamental effectors, thus offering an ideal surrounding to the cells. With this aim, different HF membrane bioreactors have been adapted and their use revised to boost the differentiation of progenitor or stem cells toward the hepatic lineage (Table 1). Monga et al. [2005] investigated the use of fetal hepatocytes as an alternative cell source in membrane bioreactor. The mentioned device was composed of 3 independent sets of HF mem-

Fibers for Bioartificial Liver and Neuronal

Tissue Engineering branes assembled according to an interwoven arrangement with the formation of a dense network. The tight and repetitive membrane units' disposition gave rise to 4 compartments with different functions. The perfusion of the fresh media was carried out by 2 sets of hydrophilic and microporous HF membranes, organized according to a countercurrent flow configuration and made up of PES with a molecular weight cut-off of approximately $500 \mathrm{kDa}$ (inner diameter $300 \mu \mathrm{m} \pm 40 \mu \mathrm{m}$; wall thickness $100 \mu \mathrm{m} \pm$ $25 \mu \mathrm{m}$; pore size $0.5 \mu \mathrm{m} \pm 0.1 \mu \mathrm{m})$. The third bundle was made up of hydrophobic multilaminate HF membrane capillaries (inner diameter $200 \pm 10 \mu \mathrm{m}$; wall thickness 42 $\pm 3 \mu \mathrm{m}$ ) for a proper gas supply, ensuring cell oxygenation and $\mathrm{CO}_{2}$ removal. The extracapillary space hosted the fetal hepatocytes that were exposed to an appropriate mass and gas exchange thanks to a membrane arrangement that mimicked the hepatic vasculature. Fetal hepatic cells within the bioreactor acquired liver-specific functions and formed a liver tissue like between the HFs, where the formation of ribbon-like structures was also visible. The overall results highlighted how the system enabled differentiation but was able to keep also an undifferentiated population. The balance between differentiation and proliferation holds the potential of solving hepatic source issues [Monga et al., 2005]. The same 4-compartment membrane bioreactor was used to study its potential to enhance hepatic differentiation of human embryonic stem cells (hESCs) in comparison with traditional culture models. The authors combined a stepwise differentiation protocol with the 3D dynamic perfusion system of the membrane bioreactor; they further established a twin bioreactor setup in which 2 devices were acting under the same operational setting but differing for the media composition. The twin approach had the aim to highlight the effects of the $3 \mathrm{D}$ perfusion on $\mathrm{hESC}$ expansion and differentiation, as a consequence of the optimal biochemical and topological stimuli offered by the HF membrane functions. Both bioreactor systems sustained cell proliferation, but a real efficient differentiation was better achieved in cells supplied with specific cytokine under 3D fiber membrane perfusion. Although the only action of the 3D perfusion conditions did not lead to a complete induction of hepatic maturation without the use of a proper pattern of specific growth factors, the tight capillary network provided an ideal environment with a proper mass exchange. This was corroborated by the enhanced effect of the cytokine pool in terms of hepatic maturation achieved in the bioreactor compared to results obtained in the traditional 2D systems [Miki et al., 2011]. The improvements of hESC differentiation toward the hepatic lineage by using a 3D HF mem- 
brane technology was also confirmed in the study of Sivertsson et al. [2013], in which the same bioreactor assembly provided encouraging data for the derivation of functional hepatocytes from an undifferentiated cell source.

The above-mentioned bioreactor was exploited for a further challenging investigation which consisted in the cultivation of total human fetal liver cells, thus comprising hepatic progenitor, hematopoietic, and endothelial populations. The experimental set-up had the aim to study the effects of a perfusion condition on their differentiation capacity. Liver cells exposed to the recirculation of the media were metabolically active showing a good differentiation state as demonstrated by the presence of mature hepatocytes and differentiated endothelial cells at the end of the culture. The synthetic activity typical of adult hepatocytes together with the endothelial expression of genes suggested that the bioreactor with $4 \mathrm{com}$ partments represents a useful tool for the establishment of an organotypic culture system for an integrated research tool in regenerative medicine. The combined approach of the increased hepatic differentiation and enhanced gene expression of endothelial cells generated a reliable in vitro tool in liver tissue engineering [Pekor et al., 2015].

More recently, the same dynamic system was also assessed for its capacity to induce a better hepatic maturation of human induced pluripotent stem cells. The experimental outcomes actually emphasized the potential of the dynamic $3 \mathrm{D}$ culture by improving the formation of differentiated tissue in terms of morphological and functional behavior [Meier et al., 2017; Freyer et al., 2018].

Amimoto et al. [2011] developed a HF/organoid culture method to induce the differentiation of mouse embryonic stem cells toward the hepatic lineage. The experimental protocol comprised the use of $6 \mathrm{HF}$ membranes usually employed for plasma separation. Membranes were constituted of cellulose triacetate with a pore size of $0.2 \mu \mathrm{m}$ (inner diameter: $285 \mu \mathrm{m}$; outer diameter: $387 \mu \mathrm{m}$ ). Undifferentiated cells were inoculated and cultured within the lumen of the membrane. After cell loading, the membrane bundle underwent a centrifugation in order to promote cell aggregation and the formation of spheroid clusters. Inside the membrane lumen, the aggregate cells proliferated along the membrane axis and acquired liverspecific functions indicating that the HF/organoid culture is a promising approach for artificial liver device in which a sufficient differentiation rate is also achieved as demonstrated by the investigation of specific hepatic parameters [Amimoto et al., 2011].
A similar experimental paradigm was used by Matsushita et al. [2019] in which induced pluripotent stem cell (iPS) differentiation was achieved after cultivation in the lumen of HF membranes. Cells were inoculated in a bundle of $6 \mathrm{HF}$ membranes composed of PE treated with ethylene vinyl alcohol for plasma separation (inner diameter $330 \mu \mathrm{m}$; thickness $50 \mu \mathrm{m}$; pore size $0.3 \mu \mathrm{m}$ ). The methodology allowed to perform high-cell-density culture of human iPS cells, in which a consistent part of the entire cell population acquired endodermal features with a trend 2 -fold higher.

The use of a HF membrane system allows to control several specific aspects that play a pivotal role for cell growing and differentiation. Therefore, the multicompartment crossed HF membrane bioreactor, developed by De Bartolo and previously described for the long-term maintenance of adult hepatocytes [De Bartolo et al., 2009], was also used for the expansion and differentiation of rat embryonic liver cells in mature hepatocytes [Salerno et al., 2013]. The bioreactor created a homogenous environment for cell culture in which the concentrations of nutrients and metabolites were monitored and controlled, and differentiation signals were provided to the cells. Progenitor liver cells cultured into the bioreactor filled the entire membrane surface organizing themselves according to a typical 3-dimensional cord-like structure, thus resembling the liver tissue disposition. Within the membrane bioreactor cells acquired morphological and functional features typical of the mature liver cells. Indeed, cells shifted toward a polyhedral shape morphology as hallmark of differentiated liver parenchymal cells and acquired synthetic and biotransformation functions. The expression pattern profile of specific proteins provided further evidences that liver differentiation took place in the bioreactor: cells lost alpha-fetoprotein expression and gained the albumin expression which are markers of hepatoblasts and mature hepatocytes, respectively.

Considering the encouraging results and the fine tuning of the proper environmental surrounding at the cellular interface, the crossed HF bioreactor [De Bartolo et al., 2009] was used to move a step forward in stem cell differentiation approaches for the formation of in vitro functional liver tissue structures [Piscioneri et al., 2018]. The strategy of the work was to study the differentiation induction of human hepatic mesenchymal stem cells (Fig. 3). To create a more cell-friendly environment, the PES HF membrane underwent a surface modification with a thin coating layer of collagen, which ameliorated cell interaction and adhesion but did not affect the membrane properties in terms of selectivity. The preservation 


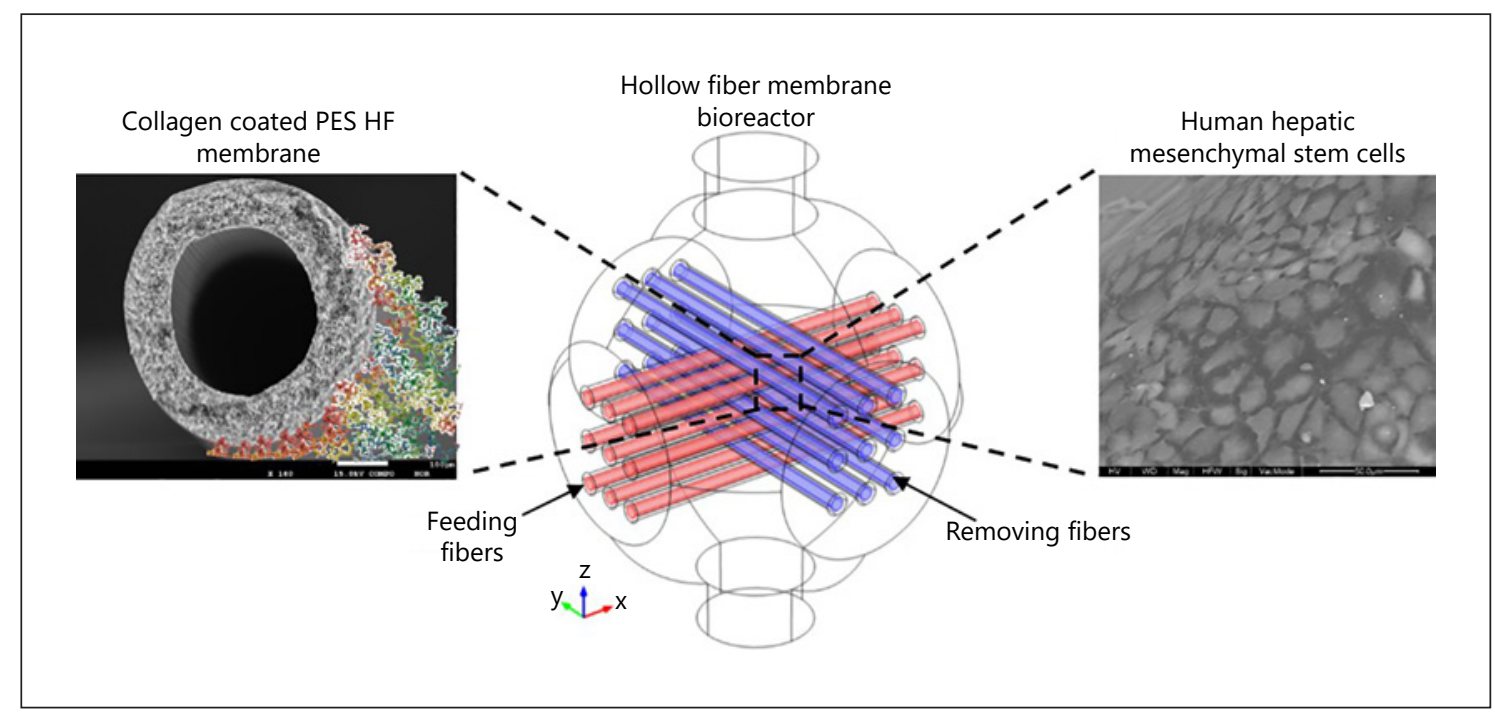

Fig. 3. Crossed modified PES hollow fiber (HF) membrane bioreactor using feeding (red) and removing fibers (blue) for the culture of human hepatic mesenchymal stem cells. Adapted from Piscioneri et al. [2018], with permission from Elsevier.

of the HF native permeability properties is quite relevant for reproducing the biomimetic delivery and exchange of oxygen and nutrients in the cell compartment, that strongly influence cell survival.

The thin collagen layer together with the intrinsic HF membrane properties offer a whole ideal set of chemical and topographical stimuli to the cells, able to promote the acquisition of morphology, polarity, and specific functionality that characterize parenchymal liver cells. The membrane bioreactor performance in terms of oxygen delivery to the cells was evaluated through cellular oxygen consumption rate measurements [Curcio et al., 2014], highlighting a proper oxygen uptake within the bioreactor chamber, avoiding the formation of any hypoxic zone. Stem cells within the membrane system went through morphological changes and were positive for specific intracellular markers of mature hepatocytes. Glycogen storage, synthetic and detoxification functions were also positively assessed confirming the acquisition of a hepatic phenotype profile.

The overall results reinforce the evidences of the useful approach of membrane technology for the realization and implementation for novel investigational platforms.

Fiber membranes can serve as self-directing biomaterials to boost liver cell production for achieving the largescale culturing and spontaneous differentiation. The huge membrane technology potential resides in its ability to establish a proper microenvironment that promotes cell adhesion and proliferation while directing the precise

Fibers for Bioartificial Liver and Neuronal Tissue Engineering acquisition of target cell genotype and phenotype as evidenced by the recent progress and emerging techniques in biomaterial-assisted cell production. Moreover, the gathered investigational studies confirm that stem cells have a great potential in the creation of bioartificial liver devices.

Improvements in terms of quality control and efficient cell differentiation within a fiber-based liver device require a stable metabolic performance together with a predictable cell behavior. These challenging issues can be faced only through the constant seeking and synergistic integration of innovative auxiliary biomaterials and high degree of differentiation methods.

\section{Nanofibers in Liver Tissue Engineering}

Tissue development within a polymeric scaffold is driven by cell-matrix interactions that primarily affect cell migration, differentiation as well as the entire cell behavior. For an efficient tissue engineered approach that provides a proper cell support, it is necessary to reproduce the natural extracellular environment. This fundamental prerequisite is satisfied by the electrospinning technique whose nanofiber production creates a biomimetic scaffold with a defined intrinsic structure, from nano- to microscale surface cues, and controllable mechanical and physico-chemical properties. Nanofibrous mats mimic the ECM milieu by providing a 3D architecture, high interconnected porosity, great absorbance, and gas permeability to the cells. Nanofiber structure, thanks 
to its disposition, promotes the interaction with integrinbinding proteins which in turn boost cell attachment and the acquisition of the appropriate morphology.

Electrospun nanofibers can be used for different tissue applications thanks to their versatile properties and enormous potential for biomedical field applications [Zhang et al., 2018; Rasouli et al., 2019]. Here, some interesting approaches about the in vitro maintenance of functional liver cells within nanofiber scaffold systems are summarized (Table 1). An example of nanofibrous mats in liver tissue engineering technology is provided by Feng et al. [2009] through the development of galactosylated chitosan (GC) nanofibers for rat primary hepatocyte culture. The bioactive scaffold, prepared by electrospinning technique, presents an average nanofiber size distribution of $160 \mathrm{~nm}$, which maintain the structural and mechanical integrity after aqueous treatment. The aim of the study was to validate the ability of GC nanofibers to enhance cell interaction and spheroid formation with the retention of specialized hepatic metabolic functions. The GC nanotopography not only enhanced high level of specific functions but also promoted a better cell-material integration when compared with flat substrates prepared from the same functionalized polymer. Indeed, on the GC nanofibers, hepatocytes formed flat spheroids firmly anchored to the nanofiber surface, while on the flat GC they can be easily removed. Spheroids formed on GC nanofibers were also metabolically more active. The study highlighted the optimal cell response to the integrated approach of nanofiber structures functionalized with galactose ligands, suggesting the great potential of nanofibrous surface for liver tissue engineering.

In another study, nanofibrous PLLA scaffolds were used for culturing primary rat hepatocytes for the realization of in vitro toxicological models [Bierwolf et al., 2011].

The scaffold was prepared through phase inversion technique giving rise to an interconnected macroporous network. The matrix architecture mimics the collagen fibrillary disposition within the ECM structure, enhancing cell seeding throughout the free space of the nanofibrous mats. The 3D scaffold well sustained hepatocyte growth which displayed a cytoskeletal structural integrity. Hepatocytes reorganized themselves along the macroporous interstices establishing cell-to-cell contacts which are fundamental for the right intercellular communication. Cells were stained for the tight junction marker ZO-1, whose visualization indicated that liver cells retained their polarized orientation. Other functionality tests as albumin production and glycogen storage corroborated the idea that PLLA nanofibrous scaf- folds create a microenvironment that preserves hepatocyte-specific functions.

An electrospun nanofibrous scaffold of PCL and chitosan was fabricated and evaluated for its potential application in liver tissue engineering [Semnani et al., 2017]. The investigation led to the realization of blended PCL/ chitosan nanofibers with excellent shape and orientation. Within the different types of nanofibers, the most suitable ones for the culture of mouse epithelial liver cells (Hepa 1-6) exhibited a diameter of $243 \pm 32 \mathrm{~nm}, 79 \%$ porosity, and an average pore size of $12 \pm 5 \mu \mathrm{m}$. The structural properties of nanofibers played a crucial role for cell colonization and infiltration, allowing the non-parenchymal liver cells to adhere along the scaffold surface. The matrix showed a good appropriateness in terms of biocompatibility and nontoxic response, validating its use for the realization of future tissue engineered liver constructs.

Hepatocyte in vitro cultures achieve successful results when defined factors are properly provided to the cells in terms of micro-environmental, physico-chemical, and mechanical stimuli. The simultaneous satisfaction of these vital factors is accomplished by using 3D scaffolds combined with ECM proteins. In this way the scaffold works as biomimetic matrix where cells can keep an in vivo-like morphology. A 3D liver tissue model for the long-term maintenance of functional hepatocytes was realized using a nanofiber scaffold made up of chitosan [Rajendran et al., 2017]. The electrospinning parameter set-up generated a highly porous scaffold with fibers randomly oriented. Structural analysis investigations evidenced a fiber diameter of 50-300 nm with an average value of $156 \pm 48 \mathrm{~nm}$. The mean pore size was estimated at $2.2 \pm 0.4 \mu \mathrm{m}$. The scaffold was colonized with a simultaneous culture of fibroblasts and hepatocytes which helps to recover intercellular contacts. Prior to cell seeding, the scaffold was coated with fibronectin to reinforce the bioactive stimulus at the cellular interface with a consequent good cellular spreading. During the culture time both fibroblasts and hepatocytes preserved their morphology, showing a wellcombined integration between them and within the scaffold mats. Cells migrated along the whole scaffold thickness as a sign of a good substrate interaction. The retention of the proper phenotype is closely correlated with the good metabolic performance of the co-cultured cells in the scaffold. Indeed, hepatocytes showed a growing albumin secretion during the whole culture time and high levels of CYP 450 A1 enzyme activity. The 3D hepatic co-culture system opened successful perspectives toward the development of nanofiber-based systems for drug testing and liver tissue engineering. 
A peculiar characteristic of the electrospun nanofiber scaffolds is the large surface area compared to the volume which is synergistically combined to a good cell adhesion and colonization and an optimized supply of nutrients. The development of innovative tissue engineering strategies implies the continuous seeking of optimized techniques which improve scaffold stability and bioactivity. In this direction, Bual et al. [2018] developed a technique aimed to improve the bioactive performance of gelatin/ PCL nanofibers blend by adding liver-specific extracellular matrix (L-ECM) derived from porcine liver in the bulk solution. Different scaffold compositions have been realized by varying the presence and the percentage of the 3 components: PCL, gelatin, and L-ECM nanofibers exhibited a smooth surface and high porosity with values ranging between 88.6 and $95.3 \%$. PCL nanofibers had a good intrinsic mechanical stability and the blending with the 2 natural components sensibly improved also its hydrophilic properties, thus ameliorating also cell interactions at its interface. The matrices were seeded with primary hepatocytes. The L-ECM-modified PCL nanofibers sustained the maintenance of a proper hepatic morphology with the formation of a tissue-like structure, where also the specific functions were carried out and maintained within the culture time. Indeed, the introduction of tissue-specific signal molecules within the polymer structure provided a balanced microenvironment to the hepatic cells which also promoted further investigations related to the effects of the different tissue-specific ECM elements for nanofiber organ constructs [Bual et al., 2018].

For hepatic culture, scaffolds with a low density fiber packing are more appropriate in order to promote cell infiltration along the whole thickness. A typical spongelike structure, highly porous and with less compact fiber structure, can be achieved through the wet electrospinning technique. Within the liver tissue engineering scenario this approach was used by Brown et al. [2018] who realized a nanofibrous scaffold made of PLGA. The resulting PLGA scaffolds were extremely porous with a mean pore size of $27.5 \pm 7.5 \mu \mathrm{m}$ (range 9.7-44.5 $\mu \mathrm{m}$ ), recapitulating the same pore size of the decellularized liver ECM and thus facilitating hepatocyte distribution along and within the scaffold. While the wet electrospinning methodology strongly affects the pore size in a significant manner, it has a minor effect on the average fiber diameter which measured $870 \pm 300 \mathrm{~nm}$.

The porous nanofibrous matrix was further improved through chemical immobilization of 2 different ECM proteins, namely collagen I and fibronectin, both used at different working concentrations. The chemical modification did not modify the overall porosity. To validate the ECM-modified nanofiber matrices for liver tissue engineering, they were used for a 14-day experiment with hepatocytes, and results were compared with the ones obtained by culturing hepatocytes within the type I collagen-Matrigel sandwich.

Coupling the larger pore size of the nanofibers with the collagen incorporation in the mates' structure improved the in vitro performance of the primary hepatocytes. Cellular state indicators of cell viability and above all the liver-specific functions were higher on cells cultured in the collagen-modified scaffold with promising future applications in the field [Brown et al., 2018].

In a similar work of Das et al. [2020], by using the same kind of membrane mentioned above, different ratios of collagen I to fibronectin were investigated and optimized in order to identify the most useful combination of the 2 proteins in sustaining hepatic cell functionality. Scaffolds after the protein chemisorption process maintained their porosity. The 3:1 ratio of collagen I to fibronectin was identified as the most suitable one, in terms of uniform distribution over the electrospun surface and the most efficient in supporting a proper liver cell functionality. Besides pointing out the importance of integrating multiple biomimetic stimuli within the structure scaffold, the study highlighted the importance of the optimal ratio of the incorporated signals to maximize cell interaction and behavior [Das et al., 2020].

In a recent work [Grant et al., 2019], the hepatic niche was successfully recapitulated in vitro by combining the electrospinning technique with the use of the full set of liver ECM proteins. The challenging approach consisted in the direct integration of the ECM proteins in the PLLA polymeric solution for the production of a bioactive electrospun scaffold. The incorporation of the entire ECM pool of proteins, derived from decellularized unused liver, was compared with integration of single ECM proteins as collagen, fibronectin, and laminin 521. The use of different proteins affected the mechanical characteristics of the scaffold. Nanofibers containing the whole ECM extract showed a Young's modulus significantly higher in comparison to the scaffold with a single protein incorporation and with the scaffold made of polymer only. This difference moves the attention to a key aspect that influences cell behavior as the material stiffness, which must be considered for the specific application [Kennedy et al., 2017]. The scaffold fabrication process retained the protein bioactivity as demonstrated by the positive staining which localized the specific antigen. The different nano- 
fibrous environments were validated using the $\mathrm{TH}-3$ liver cells, by testing their viability and adhesion. Protein scaffolds maintained both cell survival and adhesion with the lowest cell percentage detected on the polymer-only scaffold. The different cell responses indirectly confirmed protein integration within the nanofibrous mats which positively affected cell behavior. Gene expression assessments, both for liver functional genes and ECM genes, confirmed that the most performant scaffold is the one in which the whole ECM extract was solubilized, corroborating that individual ECM elements are not able to fully recapitulate the native liver environment.

A 3-dimensional micro-nano multiscale fiber-based membrane system was recently developed by Verma et al. [2018b]. The fiber-based substrate presents a peculiar double membrane arrangement: a polymeric blend of PCL, chitosan, and gelatin bead-free nanofibers have been layered all over the outer layer of PES HF membranes. The set-up membrane preparation allows the concurrent combination of 2 membrane configurations, besides the advantage to have also the simultaneous presence of different polymers, whose appropriate combination allows to reach optimal membrane properties. The deposition of nanofibers on PES HF membrane improved its mechanical properties. Thanks to the large surface area of the outer nanofibers together with their increased ECM mimicking activity and the combination of 3 different bioactive polymers, the substrate showed great attitude for its use in the realization of bioartificial liver device. Indeed, HepG2 cells cultured on the nanomicro multiscale membrane, adhered over the surface maintaining also an efficient functionality, validating its further use for liver tissue engineering [Verma et al., 2018b].

The common feature of all these systems is their great promise in representing valuable tissue models for future tissue engineered applications and patient treatment, not only in the field of liver but also for a wide variety of tissues and organs being easily adaptable for different purposes.

\section{Fiber-Based Approaches for Neuronal Tissue Applications}

Biomedical engineering approaches combine the efforts of biologists, engineers, and material scientists to develop a diversity of multifunctional devices to understand, treat, and finally solve the nervous system damages and disorders. Current strategies for repairing damaged neurons in the peripheral and central nervous system, after traumatic injuries or disease, have focused on developing artificial materials to enhance the regenerative abilities of neurons [Cangellaris and Gillette, 2018]. In this context, decades of advances in membrane technology have led to its application in neuroscience and neural engineering. Several papers indicated that membrane-based systems provide in vitro advanced devices that enhance neuronal growth and differentiation and enable the repair and regeneration of the nervous system [De Bartolo et al., 2008; Morelli et al., 2010, 2015b, 2017a]. In addition, neuronal biohybrid membrane systems can mimic specific features of the in vivo neuronal environment and, therefore, are also widely used as in vitro brain tissue models for pharmacological screening and as investigational platform for neurodegenerative diseases [Giusi et al., 2009; Morelli et al., 2014, 2016, 2019, 2021; Piscioneri et al., 2015, 2021; Mele et al., 2017].

Autologous nerve grafting, the gold standard method for the treatment of peripheral nerve injury, is associated with various complications including neuroma, donor site morbidity, and limited amounts of donor tissue. Tissue engineering studies indicate that synthetic nerve guide conduits (NGCs) represent a promising alternative for peripheral nerve repair, overcoming the limitations of autografts.

An ideal NGC must be biocompatible, biodegradable, soft and flexible, and semipermeable, which can provide a guidance cue via $3 \mathrm{D}$ tubular structure, prevents fibrous tissue from ingrowth, and meets technical requirements for further production, sterilization, long-term storage, and surgical handling [Kehoe et al., 2012]. NGC not only can give a support for axon regeneration, but also can guide the axons' regeneration and construct a permissive microenvironment for nerve regeneration.

Among the different biomaterials currently used as NGC, polymeric membranes in fiber configuration show great potential for nerve regrowth and reconstruction of nerve functions. HF membranes compartmentalize axonal processes from their cell bodies and can enable neuronal cultures with directed neurite outgrowth within a 3D space for controlling neuronal cell networking. Such kind of fiber device can provide neurotrophic support for injured nerves, retard the fibrous tissue infiltration, and guide regenerating axons to appropriate targets, and could be as effective as autografts [Yu and Bellamkoda, 2003].

Fiber membranes used for neuronal bridging devices include nondegradable and degradable materials (Table 1). The first example of HF membrane application in 


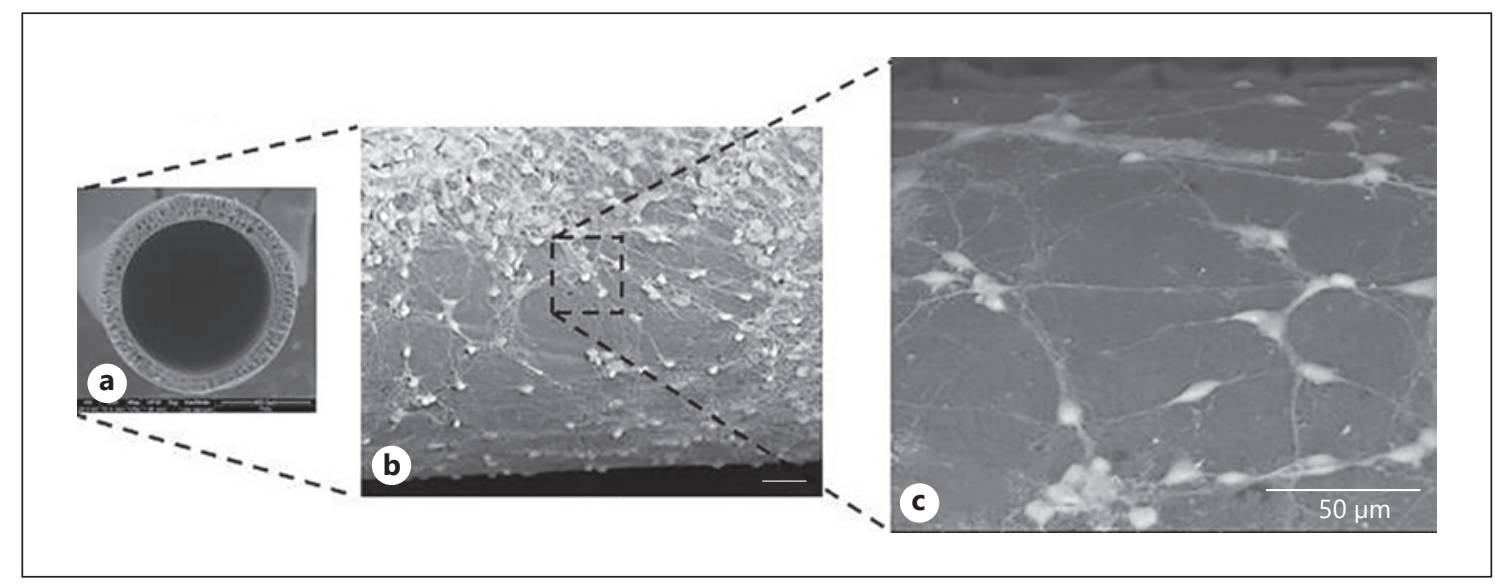

Fig. 4. Hippocampal neurons cultured on PAN hollow fiber membranes. SEM micrographs of the cross section of membranes (a) and hippocampal neurons (b, c) after 12 days of culture. Adapted from Morelli et al. 2012a and $2012 \mathrm{~b}$, with permission from Springer and Wiley, respectively.

central nervous system tissue was made of nondegradable acrylic copolymer, the Diaflo HF membrane microdialysis tube fabricated by Benveniste and Diemer [1987] and implanted in the rat hippocampus. Porous tube in nondegradable poly (2-hydroxyethyl methacrylate-co-methyl methacrylate), realized by Reynolds et al. [2008], was capable of improving locomotion functions in rat after spinal cord transection surgery. Another nondegradable polymer, PSf, was used to prepare semipermeable HF membranes with MWCO of $100 \mathrm{kDa}$ that supported the regeneration of hamster sciatic nerve [Aebischer et al., 1989].

Brayfield et al. [2008] utilized excimer laser ablation to generate specifically sized channels within the walls of PES HFs in order to compartmentalize growth of neuronal cell bodies from their axonal processes and further facilitate directed process growth into the $3 \mathrm{D}$ space of the fiber lumens. They incorporated these materials into a bioreactor to establish high-density 3D in vitro neuronal cell cultures with defined axonal pathways in the directions of the lumens of the scaffolds to create a tool for studying $3 \mathrm{D}$ neuronal networking in vitro. PES HF membranes were also used for encapsulating glial cell line-derived neurotrophic factor for the regeneration of the nigrostriatal dopaminergic fibers in an animal model of Parkinson disease [Sajadi et al., 2006].

HF membranes made of nondegradable polyacrylonitrile and polyvinyl chloride copolymer (PAN-PVC) were used to encapsulate PC12 cells secreting L-dopa. Broadhead et al. [2002] examined the influence of PAN-PVC HF membrane transport properties on encapsulated PC12 cell behavior. The study indicated that encapsulat- ed cell biomass, the number of proliferating cells, and the quantity of dopamine release increased as a function of increasing HF membrane diffusive permeability.

A selective permeability is desired to allow the free transport of vital elements, such as nutrients, growth factors, and oxygen to the cells, while excluding large immunogenic or inhibitory molecules and metabolic products from the cellular environment. Therefore, transport properties of fiber membranes play a crucial role in driving neuronal growth and axonal regeneration. In this context, PAN and modified PEEK-WC HF membranes were utilized to evaluate the influence of membrane permeability on neuronal behavior [Morelli et al., 2012a]. Hippocampal neurons respond to the different HF membranes by regulating their morphology and changing their axonal growth and metabolic functions. PAN HF membranes induced better neuronal outgrowth with a more functional and complex neuronal network than the PEEK-WC ones (Fig. 4). These differences are due to the PAN membrane intrinsic geometry and higher hydraulic permanence, which enhance the mass transfer of nutrients and metabolites to the cells and the removal of catabolites. This study confirmed the correlation of the membrane properties with cellular responses, especially the key role of membrane permeability in controlling cellular microenvironment by regulating the supply of nutrients and gases [Morelli et al., 2012b]. Therefore, this aspect must be considered in designing valuable biomaterials for neuronal tissue engineering.

Despite the many successful uses of the nerve conduits prepared from nondegradable biomaterials, these systems remain in situ as a foreign body, causing a chronic tissue 
response, nerve compression, and infection. A secondary surgery is required to remove it and circumvent these problems. To overcome these drawbacks, recent research has been focused on the production of artificial nerve guides made with biodegradable polymers, which after serving their function, gradually degrade within a reasonable period without inducing an adverse tissue reaction.

Several types of natural and synthetic biodegradable polymers including collagen and hyaluronic acid, PLGA, PU, and PCL have been fabricated into fiber structures for axonal guidance purposes.

PCL melt-extruded membranes were effective for the repair of small $(0.5 \mathrm{~cm})$ and medium $(1.5 \mathrm{~cm})$ size nerve defects in the peroneal and median nerve of Wistar rats [Chiono et al., 2009].

The importance of wall porosity on the regeneration process is noteworthy, and then different strategies and approaches have been introduced to enhance porosity and pore interconnectivity of novel nerve conduits. Oh et al. [2008] prepared asymmetrical porous fibers of PLGA and Pluronic F127 with selective permeability and hydrophilicity as NGCs by non-solvent-induced phase separation in order to produce materials with interconnected porous structure. The inner surface of the fibers had nano-size pores which can effectively prevent fibrous tissue infiltration but permeate nutrients and retain neurotrophic factors, while the outer surface had micro-size pores which allow vascular ingrowth for effective supply of nutrients into the tube. In vivo studies in rat sciatic nerve demonstrated that PLGA/F127 membranes showed better nerve regeneration behavior than the non-porous control silicone or hydrophobic PLGA tubes.

Chitosan, a natural bioactive biopolymer, with excellent properties of low toxicity, antibacterial properties, high biocompatibility and biodegradability, has been commonly employed for fabricating fiber membranes as guidance conduit for the proliferation and differentiation of neuronal cells. Wang et al. [2007] realized porous chitosan nerve conduits reinforced by braided chitosan fibers by a combination of the mold casting and the lyophilization technique. The reinforced guide conduit had sufficient permeability that allowed penetration of molecules ranging in size from 180 to $66,200 \mathrm{Da}$. In vivo and in vitro studies indicated that the chitosan fibers improve the biocompatibility and mechanical properties of the guide conduit and make the reinforced material fulfill the requirements of nerve regeneration.

Recently, a novel composite chitosan-based nerve graft comprising microporous chitin-based conduit and internal chitosan fibers was constructed and applied to bridge sciatic nerve across a $10-\mathrm{mm}$ defect in rats. In this study, chitin-based hollow conduit was fabricated by the solution coating method, and chitosan fibers were placed in the lumen of the conduit serving as internal filler to guide the growth of nerve cells. The composite artificial nerve graft had suitable mechanical strength, porosity, inner diameter, and wall thickness. The nerve graft possessed good in vitro and in vivo biocompatibility and biodegradability [Jiang et al., 2019].

In addition to the material property of neural conduit, the structure and surface topography of membranes are used for directing neurite outgrowth and are useful topics in nerve tissue engineering to devise methods for improving nerve regeneration in vivo. Zhang et al. [2005] created aligned grooves on the inner surfaces of HF membranes, providing guidance channels for the orientation and the directional outgrowth of regenerating axons.

\section{Advanced Designs of Fiber Conduits for Nerve \\ Guidance}

To date, much progress has been made in all the aspects of making nerve guidance conduits including the design, materials, and fabrication techniques. Various spinning methods have been developed to produce micro- or nanoscale fibers, and the most commonly used methods among them are electrospinning and microfluidic spinning that can control the shape, surface features, and chemical composition of a single fiber. The $2 \mathrm{D}$ and 3D materials consisting of these fibers can provide chemical and physical cues to regulate neuronal behaviors including cell adhesion, proliferation, morphogenesis, and differentiation.

The success of electrospun fibers in the tissue engineering field resides in their capacity to offer the cells an environment that mimics the native ECM.

In vitro studies have reported on the development of electrospun fibers with a random orientation that could be employed as neuronal conduits.

Randomly oriented electrospun nanofibers of glycidoxypropyltrimethoxysilane were easily fabricated by electrospinning and supported the adhesion and proliferation of glial-like cells [Tonda-Turo et al., 2013].

Chen et al. [2011] designed cationic chitosan-graftPCL/PCL hybrid electrospun nanofibers with random orientation for retinal tissue engineering. This material was characterized by high hydrophilic properties, and diameters ranged from $656 \pm 53 \mathrm{~nm}$ to $925 \pm 42 \mathrm{~nm}$ without bead formation, which favored the proliferation and differentiation of retinal progenitor cells toward neuronal lineage. 
Another kind of randomly oriented electrospun nanofibrous membranes was prepared by Zhang et al. [2015] by blending silk fibroin and poly(L-lactic acid-co- $\varepsilon$ caprolactone) for retinal progenitor cell proliferation and differentiation.

Since the native peripheral nerve bundles are comprised of highly ordered neuronal cell-matrix assemblies, and the nano/submicron alignment of fibrous matrix plays an important role in the orientation of cellular growth, unidirectional aligned electrospun fibers are of great potential in nerve regeneration. The orientation of the fibers can direct the outgrowth of neurons, providing topographical guidance for the regeneration of axons and enhancing the diffusion of neurotrophic factors. The importance of micro- or nanofiber alignment for directing the growth of neural stem cells, neurons, and glial cells has been demonstrated through testing many aligned electrospun fiber membranes made of both natural and synthetic polymers [Yang et al., 2005; Lim et al., 2010; Wang et al., 2010; Cooper et al., 2011; Subramanian et al., 2012; Das et al., 2015].

Electrospun chitosan nanofibers showed particularly high potential for nerve tissue engineering. Schwann cells and PC12 cells grew highly oriented on the aligned chitosan-PCL fibers by following fiber alignment direction. Additionally, neurons showed enhanced upregulation of differentiation-specific gene expressions [Cooper et al., 2011].

Zamani et al. [2014] showed that PLGA-aligned nanofiber membranes enhanced the axonal regeneration in a transected spinal cord rat model. Despite the positive results, only limited studies have been conducted using electrospun fibers in spinal cord tissue engineering.

Many changes have been made in the electrospinning process to achieve aligned fibers that serve as guidance conduits for directing neuron alignment. Jha et al. [2011] used two pole air gap electrospinning to fabricate $3 \mathrm{D} \mathrm{cy-}$ lindrical constructs composed of PCL fibers with nanoto-micron diameter, which have been deposited into highly aligned arrays oriented parallel to the long axis of the constructs. The architecture of these constructs provides thousands of potential channels that reconstructed 10 -mm lesions in the rodent sciatic nerve.

Shih et al. [2012] used the bioelectrospinning method for realizing PLLA-aligned fibers incorporating cells and growth factors. This novel approach reduced the dimension of the guiding structural unit for maximum cell attachment and growth, but also effectively integrated all 3 major components of tissue engineering into one simple low-cost process.

Fibers for Bioartificial Liver and Neuronal Tissue Engineering
A novel advanced neuronal conduit device was realized by integrating PLLA highly aligned and packed micron-scale microtube array (MTA) membranes within a bioreactor that served as dynamic system to create a wellcontrolled microenvironment for neuronal growth [Morelli et al., 2017b]. PLLA-MTA membranes were prepared by a co-axial electrospinning process and besides a peculiar disposition possess a porous wall structure. The continuous one-by-one HF array structured along a single layer, provided a high surface area for cell adhesion. The overall structure gave rise to a 3D neuronal-like construct by combining in vitro culture of neuronal cells and PLLA-MTA membranes in a fully controlled bioreactor. The PLLA-MTA membrane bioreactor supported neuronal growth and differentiation and allowed the long-term maintenance of specific differentiated features. Neurites linearly extended along the membranes and the elongation of neuronal processes followed the orientation and the alignment of the membranes.

Several papers have reported that the combination of different cues including topographic, biochemical, and electrical stimuli, could improve nerve regeneration and functional recovery. The association of topographic and biochemical cues by encapsulating neural growth factors into the fibers can promote greater functional recovery of the peripheral nerves. Wang et al. [2012] compared aligned PLGA nanofibers with aligned PLGA nanofibers containing nerve growth factor (NGF) in their core for the regeneration of rat sciatic nerve defect. The study demonstrated that the combined effect of physical guidance cues and biomolecular signals provided by the PLGA-NGF fibers improved the peripheral nerve regeneration.

In addition to the imitation of native nerve structure, research on conductive polymers and electrical stimulation emerges as a relatively novel approach to increase neurite extension and axonal regeneration. Conductive biomaterials that are able to enhance neurite extension with low electrical stimulation, such as polypyrrole (PPy), polyaniline (PANi), and $\beta$-tricalcium phosphate $(\beta-\mathrm{TCP})$, have been applied for the creation of nerve conduits. Aligned PLGA nanofiber coated with PPy supported the growth and differentiation of rat PC12 cells and hippocampal neurons [Lee et al., 2009]. PLLA-PANi scaffolds directed nerve stem cell differentiation [Prabhakaran et al., 2011]. A chitosan nanofiber mesh tube, on which electrically polarized $\beta$-TCP particles were immobilized, was employed to perform bridge grafting into sciatic nerve defects in Wistar rats. This approach created a suitable nerve conduit that induced functional recovery of both

Cells Tissues Organs 2022;211:447-476 467 
motor and sensory nerves after implantation, comparable with isografting [Wang et al., 2010]. More recently, other electrically conductive materials such as gold nanoparticles were incorporated into aligned electrospun silk fibroin/PEO nanofibers, and the resulting nanofiber-based nerve conduit promoted adhesion and proliferation of Schwann cells in vitro and showed good biocompatibility with surrounding tissue in vivo [Cooper et al., 2011].

The failure of nerve conduits could be attributed to luminal volume decrease, suture pull-out with traction during movement, conduit collapse, and swelling caused by material overdose. These problems can be solved by increasing the mechanical strength of the conduits and greatly improving the kink resistance while using less biomaterial. Quan et al. [2019] designed a new style of NGC with a helix-flexible structure, which was prepared using a simple electrospinning manufacturing process, and compared its mechanical properties and flexibility with those of conventional nerve conduits in order to address the problems of conventional conduits often mentioned in clinical feedback. In vivo tests in a rat sciatic nerve defect model indicated that this kind of helix-flexible nerve conduit could be used to repair peripheral nerve damage in a cross joint region with less tension during operation and easy postoperative rehabilitation.

Different approaches have been combined to realize advanced and very complex multi-structural nerve conduits (Table 1). Aligned electrospun fibers were placed on the surface of porous membranes and rolled and longitudinally glued that successfully regenerated injured nerve [Okamoto et al., 2010; Yucel et al., 2010].

Multi-channel design with external porous wall and intraluminal channels was a popular approach to design multi-structural NGCs. One of the first multiple-channel NGCs was prepared by injection molding with rapid solvent evaporation method that successfully promoted spinal cord axon regeneration [Moore et al., 2006].

A method combining injection molding and thermally induced phase separation techniques was developed to create multiple-channeled nanofibrous membranes that provided a beneficial luminal microenvironment that enhanced the attachment of PC12 rat neuronal cells, guided axonal orientation and regeneration [Sun et al., 2012] and successful differentiation of nerve stem cells into neurons [Zeng et al., 2014]. Jeffries and Wang [2013] used electrospinning to fabricate aligned fibrous mats and rolled them up to multi-channel NGCs. The advantage of this process compared to the ones fabricated using injection molding is the wall-thickness reduction, providing a greater area for tissue growth. In vitro studies demon- strated efficient cell infiltration and axonal elongation. A drawback of the fabrication method is its labor intensity and the poor repeatability and reproducibility.

Bi-layer electrospun nanofibrous nerve conduits with a combination of randomly oriented and aligned fibers were designed such that the aligned fibers on the inner layer provided topographical cues for neural alignment, while the randomly oriented fibrous outer surface provided mechanical strength to the scaffold [Panseri et al., 2008; Zhu et al., 2011]. Kim et al. [2016] made a slight modification of the bi-layer scaffold design. The inner surface was covered with highly aligned electrospun nanofibers to enhance the proliferation of neural cells, the central part of the tube was double-coated with randomly oriented nanofibers over the aligned ones, strengthening the weak mechanical strength of the aligned nanofibers.

Huang et al. [2015] prepared by electrospinning a novel fibrous conduit consisting of cellulose acetate butyrate $(\mathrm{CAB})$ longitudinally aligned nanofibers with longitudinal nanogrooves on the fiber surface. In vivo results on rats using a sciatic nerve injury model indicated that the $\mathrm{CAB}$ fibrous conduits, thanks to the highly ordered secondary structure formed by surface grooves and an increase in the specific surface area, considerably enhanced nerve repair and regeneration.

Over the last decade, microfluidic technology has emerged as an advanced method for fabricating fibers at micro- and nano-scale, which are of high interest for biomedical applications, especially for the use in peripheral nerve repair. In a recent study, Haynl et al. [2016] produced by microfluidics highly aligned collagen fibers yielding minimum dry diameters of only $3 \mu \mathrm{m}$ that allowed neuronal cell adhesion. Due to the low diameters and to the high tensile strength and Young's modulus, which exceeded that of fibers produced in classical wetspinning devices, this kind of fibers provided cell alignment and axon growth along the microfiber axes inaugurating potential applications in peripheral nerve repair.

Wei et al. [2018] combined a facile capillary-based microfluidic device and a rotary receiving pool to fabricate collagen hydrogel microfibers with highly oriented structure. In vitro culture suggested that these microfibers guided neural cell alignment along with fiber axon and promoted neuronal functions. Endothelial cells were introduced to construct a co-cultured microfiber model, which further facilitated the functional expression of neural cells due to the synergistic effect of both vascularizedlike cells and neural-like cells. This approach not only has potential to engineer complex 3D tissues with highly ori- 
ented features, but also is effective in creating pre-vascularized tissue constructs.

An important limitation of commercially available NGCs is the length of the defect that can be treated and the lack of a suitable internal guidance. A recent study by Pawar et al. [2019] investigated a novel nerve conduit made of a recombinant spider silk nonwoven mesh tube that ensures structural integrity of enclosed, microfluidics-produced collagen fibers, allowing guidance of neuronal cells and their neurites and at the same time allows for sufficient nutrient exchange. The combination of both materials is highly beneficial, because the collagen fibers allow high neurite alignment and a biomimetic environment, whereas the nonwoven mesh tube ensures the structural integrity of the collagen fibers.

\section{Fiber-Based Tools to Model Neurodegenerative}

\section{Disease and Blood-Brain Barrier}

Current challenges in neuronal tissue engineering include the creation of in vitro membrane-based models of brain tissue by combining neurons, membranes, and therapeutic molecules, which could be used as innovative approaches to investigate specific biological phenomena and/or pathological states. The main goal is to realize valuable models that can predict the results of in vivo studies and provide deriving therapeutic strategies enhancing nerve regeneration.

To date, several examples of fiber membrane-based tools have been employed as in vitro models of neurological diseases and blood-brain barrier (BBB), for testing pharmaceutical compounds in neurodegenerative diseases, and for studying the transport of therapeutic agents across the $\mathrm{BBB}$ together with $\mathrm{BBB}$ functions and properties, respectively (Table 1).

Recent improvements in cell cultures and membrane technology, such as bioreactor-based approaches, have led to the creation of advanced neuronal membrane systems, which provide investigational platforms for neurodegenerative diseases. Two different kinds of new membrane devices were developed for creating neuronal tissue engineered models for Alzheimer disease treatment [Morelli et al., 2016, 2019]. More specifically, one bioreactor comprised the use of PAN HF membranes [Morelli et al., 2016], and the other one consisted of highly aligned microfibers of PLLA prepared via electrospinning [Morelli et al., 2019]. In both cases, the fibers were assembled within a chamber in order to establish intraluminal compartments for medium flow and an extraluminal one for neuronal culture that communicate through the pores of the membrane walls. The low shear stress and 3D homogenous and stable

Fibers for Bioartificial Liver and Neuronal

Tissue Engineering in vitro microenvironment within these membrane bioreactors provided optimal conditions for the growth and differentiation of neural cells, as well as for the long-term maintenance of their metabolic functions. The creation of a $3 \mathrm{D}$ and functional neuronal tissue-engineered system was successfully achieved in vitro within both systems. Therefore, a PAN HF bioreactor and electospun PLLA fiber bioreactor were used as distinct in vitro models of $A \beta$ induced toxicity associated with Alzheimer disease to test the neuroprotective effect of carotenoid crocin, and isoflavone glycitein, respectively. Crocin inhibited the aggregation of $A \beta$ that is responsible for the onset of neurodegenerative events associated with Alzheimer disease and thus protected neurons against $A \beta$-induced neurotoxicity [Morelli et al., 2016]. Also glycitein protected neurons from the events induced by $\beta$-amyloid aggregation, such as the production of ROS and the activation of apoptotic markers, and ensured the viability and maintenance of neuronal functions (Fig. 5) [Morelli et al., 2019]. Such kind of membrane bioreactors have great potential as investigational tools in preclinical research, contributing to expand the available in vitro devices for drug screening.

During the last decades different kinds of BBB models have been realized by using different approaches, including static and dynamic platforms, as well as different cell types (astrocytes, pericytes, and endothelial cells), in order to mimic the growth environment of in vivo brain and the physiological environment of microvascular endothelial cells [Stanness et al., 1997; Parkinson et al., 2003; Cucullo et al., 2007]. In addition, cell cultures for BBB modeling have grown in structural complexity ranging from basic monocultures to multiple culture systems such as double and triple cocultures.

The most advanced in vitro BBB models are based on the use of dynamic HF apparatus characterized by a $3 \mathrm{D}$ architecture employed to explore BBB functions and to test the permeability and potential efficacy of drugs targeting the brain.

The functional core of the apparatus is represented by a bundle of microporous PP HFs, which enable coculturing of endothelial cells from various origins (animal or human, both primary cultures and cell lines) with astrocytes. Endothelial cells are seeded intraluminally in the HF membranes, whereas astrocytes are cultured on the extraluminal surface. The HFs are connected to a perfusion system that allows gas and nutrient exchange under the exposure of quasi-physiological pulsatile laminar flow. This provides the formation of a BBB that closely resembles the in situ one both functionally and anatomically [Stanness et al., 1997; Cucullo et al., 2002] and allows 


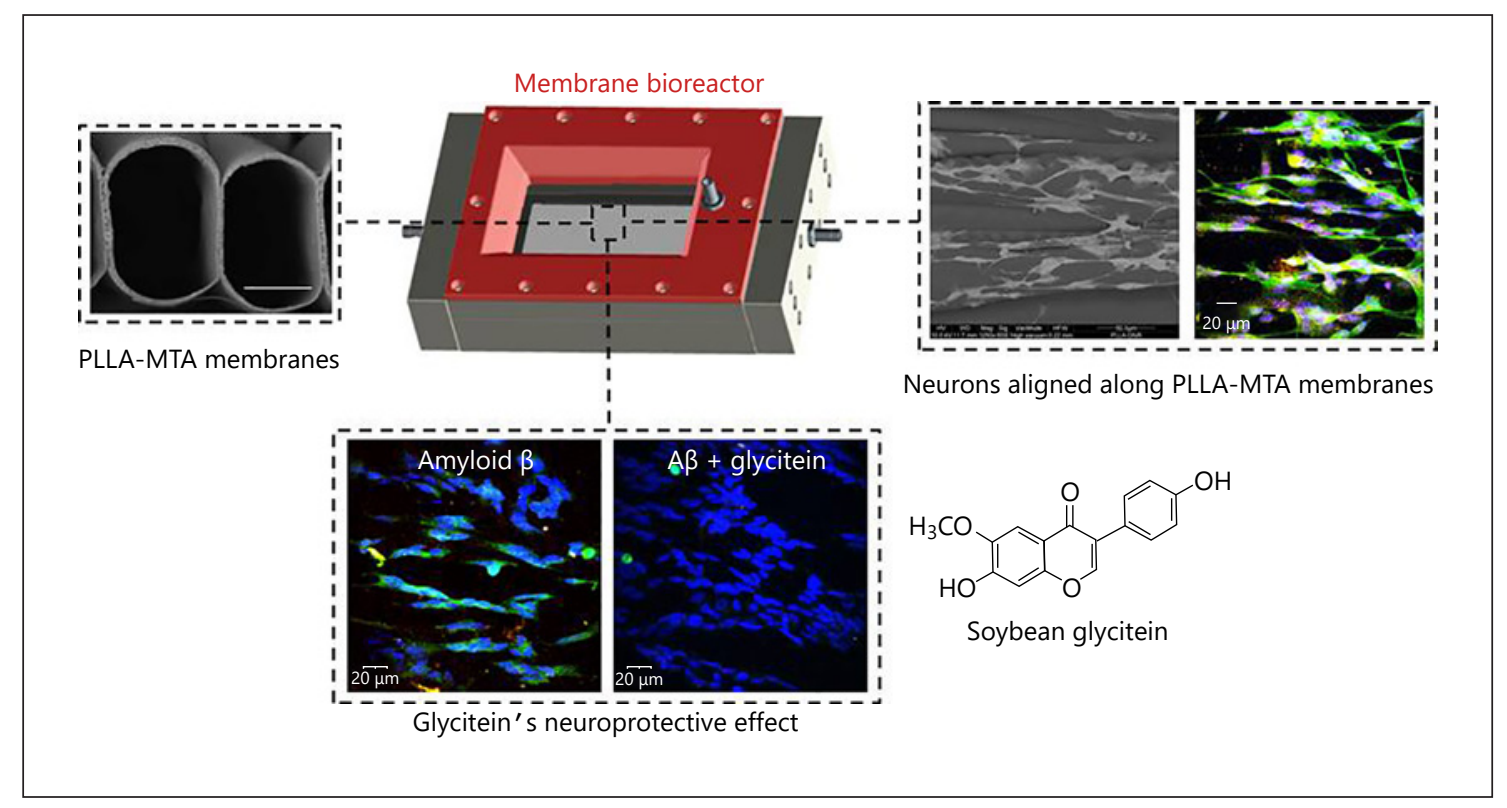

Fig. 5. Neuronal PLLA-MTA membrane bioreactor as investigational tool to test the neuroprotective effect of isoflavone glycitein against $\mathrm{A} \beta$-induced toxicity associated with Alzheimer disease. Reprinted from Morelli et al. [2019] with permission from Elsevier.

reproduction of multiple $\mathrm{BBB}$ properties that can help to address several general BBB questions. This HF dynamic $\mathrm{BBB}$ model has been used to investigate the effects of flow on endothelial cell metabolism and gene expression [Cucullo et al., 2002], demonstrating low permeability to intraluminal potassium and polar molecules (e.g., sucrose and other paracellular markers), high electrical resistance, and the expression of specialized transporters and ion channels [McAllister et al., 2001; Cucullo et al., 2002; Krizanac-Bengez et al., 2003]. The systems enable to demonstrate: (1) the key role played by the cross-talk between glial and endothelial cells in maintaining a viable BBB [Stanness et al., 1997], (2) the capacity of dipyridamole, a nucleoside transport inhibitor, to decrease the permeation of total purines, especially inosine, across the barrier and also improve specific BBB functions [Parkinson et al., 2003], and (3) the brain penetration ability of an antiepileptic drug, phenytoin [Cucullo et al., 2007].

In the last few years, the field of BBB in vitro modeling has seen many radical changes with the introduction of novel technologies and methods to improve existing models and develop new ones. Microfluidics technology has improved $\mathrm{BBB}$ modeling by providing tools with more realistic dimensions and geometries, and by exposing the endothelium to physiological fluid flow, and thus enables the real-time study of cells in a 3D engineered physiological microenvironment [Booth and Kim, 2012].

\section{Current Limitations and Challenges}

The common strategies applied in bioartificial organs and tissue engineering consist in culturing specific cell types within a $3 \mathrm{D}$ environment that mimics, as closely as possible, the natural ECM. This approach involves the use of properly designed bioreactors, which are able to reproduce all necessary specific functions for cell viability and functionality. However, the formation of organized and functional tissues is a very complex task. Tailor-made membranes designed and operated according to well-defined engineering criteria can replace the vascular system of human anatomy providing adequate transport of oxygen, nutrients, and catabolites throughout the cellular compartment, and supplying appropriate biomechanical stimuli. Nowadays, the success of this approach depends on the full understanding and control of different parameters related to material properties and hydrodynamics of the cellular environment. Materials and scaffolds are mainly developed from synthetic polymers and ECM components (e.g., collagen single strands, elastin, fibronectin, and laminin) to mimic the actual ECM. Often engineered biomaterials are designed taking into account few parameters (e.g., stiffness) of the target tissue, reducing its complexity and allowing a very limited view. For example, the stiffness of materials in contact with neuronal cells is often unreliable, because the stiffness of the brain tissue at all scales, 
from bulk to nanoscale under physiological and pathological conditions, is unknown [Axpe et al., 2020]. The field must move toward considering all properties (e.g., mechanical, physical, chemical, structural, transport) of a tissue or organ at different scale and under different conditions in order to realize a biomaterial able to perform the required multiple functions for its successful use in a given application. A biomimetic approach should utilize specific molecules typical for a target tissue that reproduce the key features of the ECM as in particular multifunctional polymer systems showing advanced profiles and properties which can adapt their behavior to environmental conditions (intelligent membranes). The membrane systems should be designed to offer optimum specificity toward the respective target cells. It is still unclear how the complex hydrodynamics of the cellular environment dramatically impacts on the design of clinically useful grafts and implants. To this purpose, CFD methodology can be used to achieve a detailed description of fluid mechanics since computational tools are able to predict and control a large number of parameters that mutually influence cells, tissues, organs, and integrated systems. Applications in the field of bioreactors provide evidence that a well-controlled environment in terms of mass transport phenomena and metabolic kinetics is able to activate a specific response of the cells and to maintain cell differentiation for a long time. Advances can be achieved not only by using CFD modeling approaches that allow also more efficient multiscale predictions (computational complexity, robust solving methods for partial differential equations, approaching the problem of moving boundary conditions), but also by experimental data that contain an accurate measurement of diffusion coefficients and reaction kinetics from which models can be developed and optimized [De Bartolo et al., 2012]. Several studies accentuate the complexity of oxygen supply in different bioartificial liver systems and devices, but some crucial properties and parameters seem to be overlooked or underestimated, such as the effective diffusion coefficient, permeability and oxygen uptake rate in cells and microtissues as well as the cell distribution and intra-porosity of the tissue formed in the device. Systematic parametric studies are necessary to design a device that ensures appropriate supply of nutrients including oxygen. Different parameters such as oxygen partial pressure, perfusion rate, HF spacing, cell distribution and microtissue size, kinetics for oxygen uptake and metabolite production, porosities of microtissues and the membrane, can affect the dissolved oxygen concentration profile inside the bioreactor [Khakpour et al., 2017]. These parameters must be studied in relation to variables of the operational condi-

Fibers for Bioartificial Liver and Neuronal

Tissue Engineering tions. Furthermore, careful automation strategies are required for stable and long-term operation of the bioartificial device optimizing its fluid dynamics and transport phenomena [Naghib et al., 2018].

\section{Conclusion and Perspectives}

This review highlights the advances made in the field of bioartificial organs and tissue engineering using fiber membrane-based approaches. Special attention is given to the multifunctional role of fiber membrane systems for liver and nerve regeneration as examples of the main biomedical applications.

Membranes in fiber configuration, due to their structural properties together with the physico-chemical and mechanical characteristics, not only provide an adhesion surface for cells, but also physical, chemical, and mechanical cues that profoundly influence the behavior and fate of cells. Moreover, thanks to their tubular geometry that mimics the anatomical vascular system, fiber membranes act as modulators for nutrient and oxygen transport from the medium to the cells, and discharge waste metabolites from the cells to the medium, and offer immunoprotection functions for the organ replacement therapy.

Advances in knowledge of physiology of liver regeneration, stem cells, and 3D scaffolds for tissue engineering have led toward efficient therapies for liver failure. In this review, we propose an update on the current fiber BAL systems used as a bridge for patients with acute liver failure awaiting transplantation. An important challenge related to the development of fiber bioreactors for BAL and liver regeneration is the automation of bioreactor control. A constant monitoring of the bioreactor environment and tissue development using advanced imaging and sensing modalities is important to monitor cell fate and tissue development in the 3D environment.

Several papers indicated that fiber membrane-based systems in diverse forms of randomly aligned nanofibers, $3 \mathrm{D}$ conduit, core-shell fibers within growth factors in the core, are capable of improving the regeneration of damaged neural tissues and show great potential as NGCs for peripheral nerve repair, overcoming the limitations of autografts. Different approaches have been combined to realize advanced and very complex multi-structural nerve conduits, such as multi-channel and bi-layer nanofibrous membranes. Therefore, the combination of different cues including topographic, biochemical, and electrical stimuli, could improve nerve regeneration and functional recovery. 
Since electrospun fiber membranes show great promise and potential for application in regeneration of many types of tissue, the examples of engineered microenvironments discussed in this review can help researchers planning future studies, and led to the elucidation and development of key design criteria for synthetic niches that allow greater control over cell fate.

To date, the development of biomimetic fiber membranes and bioreactors is still very challenging and requires specific improvements to permit the clinical translation of these technologies to regenerative medicine.

The challenge for the upcoming decade of tissue regeneration research remains the creation of scaffolds with precisely controlled, tunable topography, chemistry, and stiffness, to be able to predict, direct, and control the function of cells toward the creation of fully functional and viable organs for transplantation in vivo.

Promising materials for the realization of advanced fiber-based constructs could include biopolymers, such as chitosan, hyaluronic acid, alginate, collagen, or electroconductive materials, such as PPy and PANi, or carbonbased nanomaterials, such as graphene and carbon nanotubes, which have shown great potential for new therapeutic and regenerative applications.

Further advances are focused on improving the biomaterial functionality by incorporating stem cells, neurotropic factors, and drugs. Moreover, novel fabrication techniques are being explored. Culturing cells in microfluidic devices holds great promise in terms of technology for bioreactor development. Electrosprayed micro-/ nanoparticles could be very promising drug carriers. The bioprinting technology, by which viable cells are incorpo- rated into the bioink and printed as a cell-laden system, can give great opportunities for the fabrication of advanced constructs for tissue engineering and regenerative medicine.

In the future, stem cell therapy with the support of functionalized electrospun scaffolds could be a promising therapy to cure human diseases.

It is auspicious that the combination of promising materials, bioactive molecules, drugs, stem cells, and bioprinting and microfluidic technologies can help to design a new set of bioinspired fiber systems that will be used in the near future (1) for the repair and regeneration not only of liver and brain but also for a wide variety of other tissues and organs, and (2) to model the physiology and/ or the pathology of all human tissues and organs.

\section{Conflict of Interest Statement}

The authors declare that they have no conflict of interests.

\section{Funding Sources}

The authors have no funding sources to declare.

\section{Author Contributions}

S.M. and L.D.B. designed the study and wrote the manuscript. A.P. and S.S. participated in data collection and equally contributed in preparing and writing the manuscript. All authors read and approved the final version of the manuscript.

\section{References}

Aebischer P, Guenard V, Brace S. Peripheral nerve regeneration through blind-ended semipermeable guidance channels: effect of the molecular weight cutoff. J Neurosci. 1989; 9(10):3590-5

Ahmed HMM, Salerno S, Morelli S, Giorno L, De Bartolo L. 3D liver membrane system by coculturing human hepatocytes, sinusoidal endothelial and stellate cells. Biofabrication. 2017a;9(2):025022.

Ahmed HMM, Salerno S, Piscioneri A, Khakpour S, Giorno L, De Bartolo L. Human liver microtissue spheroids in hollow fiber membrane bioreactor. Colloids Surf B Biointerfaces. 2017b;160:272-80.

Amimoto N, Mizumoto H, Nakazawa K, Ijima H, Funatsu K, Kajiwara T. An evaluation of the utility of the hepatic differentiation method using hollow fiber/organoid culture for the development of a hybrid artificial liver device. Biochem Eng J. 2011;56(1-2):69-74.

Aragón J, Salerno S, De Bartolo L, Irusta S, Mendoza G. Polymeric electrospun scaffolds for bone morphogenetic protein 2 delivery in bone tissue engineering. J Colloid Interface Sci. 2018;531:126-37.

Axpe E, Orive G, Franze K, Appel EA. Towards brain-tissue-like biomaterials. Nat Commun. 2020;11(1):3423.

Benveniste H, Diemer NH. Cellular reactions to implantation of a microdialysis tube in the rat hippocampus. Acta Neuropathol. 1987;74(3): 234-8.

Bierwolf J, Lutgehetmann M, Feng K, Erbes J, Deichmann S, Toronyi E, et al. Primary rat hepatocyte culture on 3D nanofibrous polymer scaffolds for toxicology and pharmaceutical research. Biotechnol Bioeng. 2011;108(1):141-50.
Booth R, Kim H. Characterization of a microfluidic in vitro model of the blood-brain barrier ( $\mu$ BBB). Lab Chip. 2012;12(10):1784-92.

Brayfield CA, Marra KG, Leonard JP, Tracy Cui $\mathrm{X}$, Gerlach JC. Excimer laser channel creation in polyethersulfone hollow fibers for compartmentalized in vitro neuronal cell culture scaffolds. Acta Biomater. 2008;4(2):244-55.

Broadhead KW, Biran R, Tresco PA. Hollow fiber membrane diffusive permeability regulates encapsulated cell line biomass, proliferation, and small molecule release. Biomaterials. 2002;23(24):4689-99.

Brown JH, Das P, DiVito MD, Ivancic D, Tan LP, Wertheim JA. Nanofibrous PLGA electrospun scaffolds modified with type I collagen influence hepatocyte function and support viability in vitro. Acta Biomater. 2018;73:21727. 
Bual R, Kimura H, Ikegami Y, Shirakigawa N, Ijima H. Fabrication of liver-derived extracellular matrix nanofibers and functional evaluation in in vitro culture using primary hepatocytes. Materialia. 2018;4:518-28.

Cangellaris OV, Gillette MU. Biomaterials for Enhancing Neuronal Repair. Front Mater. 2018; $5: 21$.

Chen H, Fan X, Xia J, Chen P, Zhou X, Huang J, et al. Electrospun chitosan-graft-poly ( $\varepsilon$-caprolactone $) /$ poly $\quad(\varepsilon$-caprolactone $)$ nanofibrous scaffolds for retinal tissue engineering. Int J Nanomedicine. 2011;6:453-61.

Chiono V, Vozzi G, Vozzi F, Salvadori C, Dini F, Carlucci F, et al. Melt-extruded guides for peripheral nerve regeneration. Part I: poly(epsilon-caprolactone). Biomed Microdevices. 2009;11:1037-50.

Cooper A, Bhattarai N, Zhang M. Fabrication and cellular compatibility of aligned chitosanPCL fibers for nerve tissue regeneration. Carbohyd Polym. 2011;85(1):149-56.

Cucullo L, McAllister MS, Kight K, KrizanacBengez L, Marroni M, Mayberg MR, et al. A new dynamic in vitro model for the multidimensional study of astrocyte-endothelial cell interactions at the blood-brain barrier. Brain Res. 2002;951(2):243-54.

Cucullo L, Hossain M, Rapp E, Manders T, Marchi N, Janigro D. Development of a humanized in vitro blood-brain barrier model to screen for brain penetration of antiepileptic drugs. Epilepsia. 2007;48(3):505-16.

Curcio E, Piscioneri A, Salerno S, Tasselli F, Morelli S, Drioli E, et al. Human lymphocytes cultured in 3-D bioreactors: Influence of configuration on metabolite transport and reactions. Biomaterials. 2012;33(33):8296303.

Curcio E, Piscioneri A, Morelli S, Salerno S, Macchiarini P, De Bartolo L. Kinetics of oxygen uptake by cells potentially used in a tissue engineered trachea. Biomaterials. 2014;35(25): 6829-37.

Das P, DiVito MD, Wertheim JA, Tan LP. Collagen-I and fibronectin modified three-dimensional electrospun PLGA scaffolds for longterm in vitro maintenance of functional hepatocytes. Mater Sci Eng C Mater Biol Appl. 2020;111:110723.

Das S, Sharma M, Saharia D, Sarma KK, Sarma MG, Borthakur BB, et al. In vivo studies of silk based gold nano-composite conduits for functional peripheral nerve regeneration. Biomaterials. 2015;62:66-75.

De Bartolo L, Bader A, Haverich A. Review of a flat membrane bioreactor as a bioartificial liver. Ann Transplant. 2001;6(3):40-6.

De Bartolo L, Morelli S, Rende M, Campana C, Salerno S, Quintiero N, et al. Human hepatocyte morphology and functions in a multibore fiber bioreactor. Macromol Biosci. 2007a; 7(5):671-80.

De Bartolo L, Morelli S, Rende M, Salerno S, Giorno L, Lopez L, et al. Galactose derivative immobilized glow discharge processed PES membranes maintain the metabolic activity of human and pig liver cells. J Nanosci Nanotech. 2006;6(8):2344-2353.

De Bartolo L, Piscioneri A, Cotroneo G, Salerno S, Tasselli F, Campana C, et al. Human lymphocyte PEEK-WC hollow fiber membrane bioreactor. J Biotechnol. 2007b;132(1):65-74.

De Bartolo L, Rende M, Morelli S, Giusi G, Salerno $\mathrm{S}$, Piscioneri $\mathrm{A}$, et al. Influence of membrane surface properties on the growth of neuronal cells isolated from hippocampus. J Membrane Sci. 2008;325(1):139-49.

De Bartolo L, Salerno S, Curcio E, Piscioneri A, Rende M, Morelli S, et al. Human hepatocyte functions in a crossed hollow fiber membrane bioreactor. Biomaterials. 2009;30(13):253143.

De Bartolo L, Leindlein A, Hofmann D, Bader A, de Grey A, Curcio E, et al. Bio-Hybrid Organs and Tissues for Patient Therapy: a Future Vision for 2030. Chem Eng Process: Process Intensification. 2012;51:79-87.

De Bartolo L, Curcio E, Drioli E. Membrane systems for bioartificial organs and regenerative medicine. Berlin: De Gruyter; 2017.

Demetriou AA, Rozga J, Podesta L, Lepage E, Morsiani E, Moscioni AD, et al. Early Clinical Experience with a Hybrid Bioartificial Liver. Scand J Gastroenterol Suppl. 1995;208:111-7.

Demetriou AA, Brown RS, Busuttil RW, Fair J, McGuire BM, Rosenthal P, et al. Prospective, randomized, multicenter, controlled trial of a bioartificial liver in treating acute liver failure. Ann Surg. 2004;239(5):660-70.

Ding YT, Qiu YD, Chen Z, Xu QX, Zhang HY, Tang $\mathrm{Q}$, et al. The development of a new bioartificial liver and its application in 12 acute liver failure patients. World J Gastroenterol. 2003;9(4):829-32.

Drioli E, De Bartolo L. Membrane bioreactor for cell tissues and organoids. Artif Organs. 2006; 30(10):793-802.

Feng ZQ, Chu X, Huang NP, Wang T, Wang Y, Shi X, et al. The effect of nanofibrous galactosylated chitosan scaffolds on the formation of rat primary hepatocyte aggregates and the maintenance of liver function. Biomaterials. 2009;30(14):2753-63.

Freyer N, Greuel S, Knöspel F, Gerstmann F, Storch L, Damm G, et al. Microscale 3D liver bioreactor for in vitro hepatotoxicity testing under perfusion conditions. Bioengineering. 2018;5(1):24.

Gan JH, Zhou XQ, Qin AL, Luo EP, Zhao WF, Yu $\mathrm{H}$, et al. Hybrid artificial liver support system for treatment of severe liver failure. World J Gastroenterol. 2005;11(6):890-4.

Gerlach JC, Encke J, Hole O, Müller C, Ryan CJ, Neuhaus P. Bioreactor for a larger scale hepatocyte in vitro perfusion. Transplantation. 1994;58(9):984-8.

Giusi G, Facciolo RM, Rende M, Alò R, Di Vito A, Salerno S, et al. Distinct alpha subunits of the GABAA receptor are responsible for early hippocampal silent neuron-related activities. Hippocampus. 2009;19(11):1103-14.

Grant R, Hallett J, Forbes S, Hay D, Callanan A. Blended electrospinning with human liver ex- tracellular matrix for engineering new hepatic microenvironments. Sci Rep. 2019;9(1): 6293.

Haynl C, Hofmann E, Pawar K, Förster S, Scheibel T. Microfluidics-produced collagen fibers show extraordinary mechanical properties. Nano Lett. 2016;16(9):5917-22.

Hoffmann SA, Müller-Vieira U, Biemel K, Knobeloch $\mathrm{D}$, Heydel $\mathrm{S}$, Lübberstedt $\mathrm{M}$, et al. Analysis of drug metabolism activities in a miniaturized liver cell bioreactor for use in pharmacological studies. Biotechnol Bioeng. 2012;109(12):3172-81.

Hosseini V, Maroufi NF, Saghati S, Asadi N, Darabi M, Ahmad SNS, et al. Current progress in hepatic tissue regeneration by tissue engineering. J Transl Med. 2019;17(1):383.

Ho Ye S, Watanabe J, Takai M, Iwasaki Y, Ishihara K. High functional hollow fiber membrane modified with phospholipid polymers for a liver assist bioreactor. Biomaterials. 2006;27(9):1955-62.

Huang C, Ouyang Y, Niu H, He N, Ke Q, Jin X, et al. Nerve guidance conduits from aligned nanofibers: improvement of nerve regeneration through longitudinal nanogrooves on a fiber surface. ACS Appl Mater Interfaces. 2015;7(13):7189-96.

Jasmund I, Langsch A, Simmoteit R, Bader A. Cultivation of Primary Porcine Hepatocytes in an OXY-HFB for Use as a Bioartificial Liver Device. Biotechnol Prog. 2002;18(4):83946.

Jeffries EM, Wang Y. Incorporation of parallel electrospun fibers for improved topographical guidance in 3D nerve guides. Biofabrication. 2013;5(3):035015.

Jha BS, Colello RJ, Bowman JR, Sell SA, Lee KD, Bigbee JW, et al. Two pole air gap electrospinning: fabrication of highly aligned, three-dimensional scaffolds for nerve reconstruction. Acta Biomater. 2011;7(1):203-15.

Jiang Z, Song Y, Qiao J, Yang Y, Zhang W, Liu W, et al. Rat sciatic nerve regeneration across a 10-mm defect bridged by a chitin/CM-chitosan artificial nerve graft. Int J Biol Macromol. 2019;129:997-1005.

Kehoe S, Zhang XF, Boyd D. FDA approved guidance conduits and wraps for peripheral nerve injury: a review of materials and efficacy. Injury. 2012;43(5):553-72.

Kennedy KM, Bhaw-Luximon A, Jhurry D. Cell-matrix mechanical interaction in electrospun polymeric scaffolds for tissue engineering: Implications for scaffold design and performance. Acta Biomater. 2017;50: 41-55.

Khakpour S, Di Renzo A, Curcio E, Di Maio FP, Giorno L, De Bartolo L. Oxygen transport in hollow fibre membrane bioreactors for hepatic 3D cell culture: a parametric study. J Membrane Sci. 2017;544:312-22.

Kim JI, Hwang TI, Aguilar LE, Park CH, Kim CS. A controlled design of aligned and random nanofibers for $3 \mathrm{D}$ bi-functionalized nerve conduits fabricated via a novel electrospinning set-up. Sci Rep. 2016;6:23761. 
Krizanac-Bengez L, Kapural M, Parkinson F, Cucullo L, Hossain M, Mayberg MR, et al. Effects of transient loss of shear stress on blood-brain barrier endothelium: role of nitric oxide and IL-6. Brain Res. 2003;977(2):239-46.

Krogh A. The number and distribution of capillaries in muscles with calculations of the oxygen pressure head necessary for supplying the tissue. J Physiol (Lond). 1919;52(6):409-15.

Lee JY, Bashur CA, Goldstein AS, Schmidt CE. Polypyrrole-coated electrospun PLGA nanofibers for neural tissue applications. Biomaterials. 2009;30(26):4325-35.

Legallais C, Kim D, Mihaila SM, Mihajlovic M, Figliuzzi M, Bonandrini B, et al. Bioengineering Organs for Blood Detoxification. Adv Healthcare Mater. 2018;7(21):1800430.

Lim SH, Liu XY, Song H, Yarema KJ, Mao HQ. The effect of nanofiber-guided cell alignment on the preferential differentiation of neural stem cells. Biomaterials. 2010;31(34):9031-9.

Lu HF, Lim WS, Zhang PC, Chia SM, Yu H, Mao HQ, et al. Galactosylated poly(vinylidene difluoride) hollow fiber bioreactor for hepatocyte culture. Tissue Eng. 2005;11(11-12): 1667-77.

Luetchford KA, Wung N, Argyle IS, Storm MP, Weston SD, Tosh D, et al. Next generation in vitro liver model design: combining a permeable polystyrene membrane with a transdifferentiated cell line. J Membrane Sci. 2018; 565:425-38.

Margulis MS, Erukhimov EA, Andreiman LA, Viksna LM. Temporary organ substitution by hemoperfusion through suspension of active donor hepatocytes in a total complex of intensive therapy in patients with acute hepatic insufficiency. Resuscitation. 1989;18(1):85-94.

Matsushita S, Kajiwara T, Mizumoto H. Expansion and differentiation of human iPS cells in a three-dimensional culture using hollow fibers and separation of the specific population by magnetic-activated cell sorting. $J$ Biosci Bioeng. 2019;128(4):480-6.

McAllister MS, Krizanac-Bengez L, Macchia F, Naftalin RJ, Pedley KC, Mayberg MR, et al. Mechanisms of glucose transport at the blood-brain barrier: an in vitro study. Brain Res. 2001;904:20-30.

Meier F, Freyer N, Brzeszczynska J, Knöspel F, Armstrong L, Lako M, et al. Hepatic differentiation of human iPSCs in different 3D models: A comparative study. Int J Mol Med. 2017; 40(6):1759-71.

Mele M, Morelli S, Fazzari G, Avolio E, Alò R, Piscioneri A, et al. Application of the co-culture membrane system pointed to a protective role of catestatin on hippocampal plus hypothalamic neurons exposed to oxygen and glucose deprivation. Mol Neurobiol. 2017;54(9): 7369-81.

Memoli B, De Bartolo L, Favia P, Morelli S, Lopez LC, Procino A, et al. Fetuin-A gene expression, synthesis and release in primary human hepatocytes cultured in a galactosylated membrane bioreactor. Biomaterials. 2007;28: 4836-4844.
Memoli B, Salerno S, Procino A, Postiglione L, Morelli S, Sirico ML, et al. A translational approach to micro-inflammation in endstage renal disease: molecular effects of low levels of interleukin-6. Clin Sci. 2010;119(4): 163-74.

Miki T, Ring A, Gerlach J. Hepatic differentiation of human embryonic stem cells is promoted by three-dimensional dynamic perfusion culture conditions. Tissue Eng Part C Methods. 2011;17(5):557-68.

Millis JM, Cronin DC, Johnson R, Conjeevaram H, Conlin C, Trevino S, et al. Initial experience with the modified extracorporeal liverassist device for patients with fulminant hepatic failure: system modifications and clinical impact. Transplantation. 2002;74(12): 1735-46.

Mizumoto H, Funatsu K. Liver Regeneration Using a Hybrid Artificial Liver Support System. Artif Organs. 2004;28(1):53-7.

Modi A, Verma SK, Bellare J. Hydrophilic ZIF-8 decorated GO nanosheets improve biocompatibility and separation performance of polyethersulfone hollow fiber membranes: a potential membrane material for bioartificial liver application. Mater Sci Eng C Mater Biol Appl. 2018;91:524-40.

Monga SP, Hout MS, Baun MJ, Micsenyi A, Muller P, Tummalapalli L, et al. Mouse fetal liver cells in artificial capillary beds in threedimensional four-compartment bioreactors. Am J Pathol. 2005;167(5):1279-92.

Moore MJ, Friedman JA, Lewellyn EB, Mantila SM, Krych AJ, Ameenuddin S, et al. Multiplechannel scaffolds to promote spinal cord axon regeneration. Biomaterials. 2006;27(3):41929

Morelli S, Salerno S, Rende M, Lopez L, Favia P, Procino A, et al. Human hepatocyte functions in a galactosylated membrane bioreactor. J Membrane Sci. 2007;302(1-2):27-35.

Morelli S, Salerno S, Piscioneri A, Papenburg BJ, Di Vito A, Giusi G, et al. Influence of micropatterned PLLA membranes on outgrowth and orientation of hippocampal neurites. Biomaterials. 2010;31(27):7000-11.

Morelli S, Piscioneri A, Salerno S, Tasselli F, Di Vito A, Giusi G, et al. PAN hollow fiber membranes elicit functional hippocampal neuronal network. J Mater Sci Mater Med. 2012a; 23(1):149-56.

Morelli S, Piscioneri A, Salerno S, Rende M, Campana $C$, Tasselli F, et al. Flat and tubular membrane systems for the reconstruction of hippocampal neuronal network. J Tissue Eng Regen Med. 2012b;6(4):299-313.

Morelli S, Piscioneri A, Salerno S, Al-Fageeh MB, Drioli E, De Bartolo L. Neuroprotective Effect of Didymin on Hydrogen Peroxide-Induced Injury in the Neuronal Membrane System. Cells Tissues Organs. 2014;199(2-3):184-200.

Morelli S, Salerno S, Holopainen J, Ritala M, De Bartolo L. Osteogenic and osteoclastogenic differentiation of co-cultured cells in polylactic acid-nanohydroxyapatite fiber scaffolds. J Biotechnol. 2015a;204:53-62.
Morelli S, Piscioneri A, Guarnieri G, Morelli A, Drioli E, De Bartolo L. Anti-neuroinflammatory effect of daidzein in human hypothalamic GnRH neurons in an in vitro membranebased model. BioFactors. 2021;47:93-111.

Morelli S, Piscioneri A, Messina A, Salerno S, AlFageeh MB, Drioli E, et al. Neuronal growth and differentiation on biodegradable membranes. J Tissue Eng Regen Med. 2015b;9(2): 106-17.

Morelli S, Salerno S, Piscioneri A, Tasselli F, Drioli E, De Bartolo L. Neuronal membrane bioreactor as a tool for testing crocin neuroprotective effect in Alzheimer's disease. Chem Eng J. 2016;305:69-78.

Morelli S, Piscioneri A, Drioli E, De Bartolo L. Neuronal differentiation modulated by polymeric membrane properties. Cells Tissues Organs. 2017a;204(3-4):164-78.

Morelli S, Piscioneri A, Salerno S, Chen CC, Chew $\mathrm{CH}$, Giorno L, et al. Microtube array membrane bioreactor promotes neuronal differentiation and orientation. Biofabrication. 2017b; 9(2):025018.

Morelli S, Piscioneri A, Curcio E, Salerno S, Chen CC, De Bartolo L. Membrane bioreactor for investigation of neurodegeneration. Mater Sci Eng C Mater Biol Appl. 2019;103:109793.

Mueller D, Tascher G, Müller-Vieira U, Knobeloch D, Nuessler AK, Zeilinger K, et al. Indepth physiological characterization of primary human hepatocytes in a 3D hollow-fiber bioreactor. J Tissue Eng Regen Med. 2011; 5(8):e207-18

Mundt A, Puhl G, Müller A, Sauer I, Müller C, Richard R, et al. A method to assess biochemical activity of liver cells during clinical application of extracorporeal hybrid liver support. Int J Artif Organs. 2008;25(6):542-8.

Nagaki M, Miki K, Kim YI, Ishiyama H, Hirahara I, Takahashi H, et al. Development and characterization of a hybrid bioartificial liver using primary hepatocytes entrapped in a basement membrane matrix. Dig Dis Sci. 2001; 46(5):1046-56.

Naghib SD, Di Maio FP, De Bartolo L, Curcio E, Di Renzo A. Automation and control system for fluid dynamic stability in hollow-fiber membrane bioreactor for cell culture. J Chem Technol Biotechnol. 2018;93(3):710-9.

Naka S, Takeshita K, Yamamoto T, Tani T, Kodama M. Bioartificial liver support system using porcine hepatocytes entrapped in a threedimensional hollow fiber module with collagen gel: an evaluation in the swine acute liver failure model. Artif Organs. 1999;23(9):822-

Nyberg SL, Shatford RA, Peshwa MV, White JG, Cerra FB, Hu WS. Evaluation of a hepatocyteentrapment hollow fiber bioreactor: A potential bioartificial liver. Biotechnol Bioeng. 1993;41(2):194-203.

Oh SH, Kim JH, Song KS, Jeon BH, Yoon JH, Seo $\mathrm{TB}$, et al. Peripheral Nerve Regeneration Within an Asymmetrically Porous PLGA/ Pluronic F127 Nerve Guide Conduit. Biomaterials. 2008;29(11):1601-9. 
Okamoto H, Hata K, Kagami H, Okada K, Ito Y, Narita Y, et al. Recovery process of sciatic nerve defect with novel bioabsorbable collagen tubes packed with collagen filaments in dogs. J Biomed Mater Res A. 2010;92(3):85968.

Panseri S, Cunha C, Lowery J, Del Carro U, Taraballi F, Amadio S, et al. Electrospun microand nanofiber tubes for functional nervous regeneration in sciatic nerve transections. BMC Biotechnol. 2008;8:39.

Parkinson FE, Friesen J, Krizanac-Bengez L, Janigro $\mathrm{D}$. Use of a three-dimensional in vitro model of the rat blood-brain barrier to assay nucleoside efflux from brain. Brain Res. 2003; 980(2):233-41

Patzer IJ, Lopez R, Zhu Y, Wang Z, Mazariegos G, Fung J. Bioartificial liver assist devices in support of patients with liver failure. Hepatobiliary Pancreat Dis Int. 2002;1:18-25.

Pawar K, Welzel G, Haynl C, Schuster S, Scheibel T. Recombinant spider silk and collagenbased nerve guidance conduits support neuronal cell differentiation and functionality in vitro. ACS Appl Bio Mater. 2019;2(11):487280.

Pekor C, Gerlach JC, Nettleship I, Schmelzer E. Induction of Hepatic and Endothelial Differentiation by Perfusion in a Three-Dimensional Cell Culture Model of Human Fetal Liver. Tissue Eng Part C Methods. 2015;21(7):70515.

Piscioneri A, Campana C, Salerno S, Morelli S, Bader A, Giordano F, et al. Biodegradable and synthetic membranes for the expansion and functional differentiation of rat embryonic liver cells. Acta Biomater. 2011;7(1): 171-9.

Piscioneri A, Morelli S, Drioli E, De Bartolo L. PLGA multiplex membrane platform for disease modelling and testing of therapeutic compounds. Membranes. 2021;11(2):112.

Piscioneri A, Morelli S, Mele M, Canonaco M, Bilotta E, Pantano P, et al. Neuroprotective effect of human mesenchymal stem cells in a compartmentalized neuronal membrane system. Acta Biomater. 2015;24:297-308.

Piscioneri A, Ahmed HMM, Morelli S, Khakpour S, Giorno L, Drioli E, et al. Membrane bioreactor to guide hepatic differentiation of human mesenchymal stem cells. J Membrane Sci. 2018;564:832-41.

Prabhakaran MP, Ghasemi-Mobarakeh L, Jin G, Ramakrishna S. Electrospun conducting polymer nanofibers and electrical stimulation of nerve stem cells. J Biosci Bioeng. 2011; 112(5):501-7.

Quan Q, Meng H, Chang B, Hong L, Li R, Liu G, et al. Novel 3-D helix-flexible nerve guide conduits repair nerve defects. Biomaterials. 2019;207:49-60.

Rajendran D, Hussain A, Yip D, Parekh A, Shrirao A, Cho CH. Long-term liver-specific functions of hepatocytes in electrospun chitosan nanofiber scaffolds coated with fibronectin. J Biomed Mater Res A. 2017;105(8):2119-28.

Rasouli R, Barhoum A, Bechelany M, Dufresne A.
Nanofibers for Biomedical and Healthcare Applications. Macromol Biosci. 2019;19(2): 1800256.

Reynolds LF, Bren MC, Wilson BC, Gibson GD, Shoichet MS, Murphy RJ. Transplantation of porous tubes following spinal cord transection improves hindlimb function in the rat. Spinal Cord. 2008;46(1):58-64.

Sajadi A, Bensadoun JC, Schneider BL, Lo Bianco C, Aebischer P. Transient striatal delivery of GDNF via encapsulated cells leads to sustained behavioral improvement in a bilateral model of Parkinson disease. Neurobiol Dis. 2006;22(1):119-29.

Salerno S, Piscioneri A, Laera S, Morelli S, Favia $\mathrm{P}$, Bader A, et al. Improved functions of human hepatocytes on $\mathrm{NH} 3$ plasma-grafted PEEK-WC-PU membranes. Biomaterials. 2009;30(26):4348-56.

Salerno S, Campana C, Morelli S, Drioli E, De Bartolo L. Human hepatocytes and endothelial cells in organotypic membrane systems. Biomaterials. 2011;32(34):8848-59.

Salerno S, Piscioneri A, Morelli S, Al-Fageeh MB, Drioli E, De Bartolo L. Membrane Bioreactor for Expansion and Differentiation of Embryonic Liver Cells. Ind Eng Chem Res. 2013; 52(31):10387-95.

Salerno S, De Bartolo L. Biohybrid membrane systems and bioreactors as tools for in vitro drug testing. Curr Pharm Des. 2017;23(2): 319-27.

Salerno S, Morelli S, De Bartolo L. Advanced membrane systems for tissue engineering. Curr Org Chem. 2017;21(17):1760-74.

Salerno S, Curcio E, Bader A, Giorno L, Drioli E, De Bartolo L. Gas permeable membrane bioreactor for the co-culture of human skin derived mesenchymal stem cells with hepatocytes and endothelial cells. J Membrane Sci. 2018;563:694-707.

Salerno S, Tasselli F, Drioli E, De Bartolo L. Poly( $\varepsilon$-caprolactone) hollow fiber membranes for the biofabrication of a vascularized human liver tissue. Membranes (Basel). 2020; 10(6):112.

Sauer IM, Gerlach JC. Modular Extracorporeal Liver Support. Artif Organs. 2002;26(8):703-

Sauer IM, Zeilinger K, Pless G, Kardassis D, Theruvath T, Pascher A, et al. Extracorporeal liver support based on primary human liver cells and albumin dialysis--treatment of a patient with primary graft non-function. J Hepatol. 2003;39(4):649-53.

Semnani D, Naghashzargar E, Hadjianfar M, Dehghan Manshadi F, Mohammadi S, Karbasi S, et al. Evaluation of PCL/chitosan electrospun nanofibers for liver tissue engineering. Int $\mathrm{J}$ Polym Mater Polym Biomater. 2017;66(3): 149-57.

Shih Y-H, Yang J-C, Li S-H, Yang W-CV, Chen C-C. Bio-electrospinning of poly(l-lactic acid) hollow fibrous membrane. Text Res J. 2012;82(6):602-12.

Shiraha H, Koide N, Hada H, Ujike K, Nakamura $\mathrm{M}$, Shinji T, et al. Improvement of serum ami- no acid profile in hepatic failure with the bioartificial liver using multicellular hepatocyte spheroids. Biotechnol Bioeng. 1996;50(4): $416-21$.

Sivertsson L, Synnergren J, Jensen J, Björquist P, Ingelman-Sundberg M. Hepatic differentiation and maturation of human embryonic stem cells cultured in a perfused three-dimensional bioreactor. Stem Cells Dev. 2013;22(4): 581-94.

Stanness KA, Westrum LE, Fornaciari E, Mascagni P, Nelson JA, Stenglein SG, et al. Morphological and functional characterization of an in vitro blood-brain barrier model. Brain Res. 1997;771(2):329-42.

Subramanian A, Krishnan UM, Sethuraman S. Fabrication, characterization and in vitro evaluation of aligned PLGA-PCL nanofibers for neural regeneration. Ann Biomed Eng. 2012;40(10):2098-110.

Sun C, Jin X, Holzwarth JM, Liu X, Hu J, Gupte $M J$, et al. Development of channeled nanofibrous scaffolds for oriented tissue engineering. Macromol Biosci. 2012;12(6):761-9.

Sussman NL, Chong MG, Koussayer T, He DE, Shang TA, Whisennand HH, et al. Reversal of fulminant hepatic failure using an extracorporeal liver assist device. Hepatology. 1992; 16(1):60-5.

Tonda-Turo C, Cipriani E, Gnavi S, Chiono V, Mattu C, Gentile P, et al. Crosslinked gelatin nanofibres: preparation, characterisation and in vitro studies using glial-like cells. Mater Sci Eng C Mater Biol Appl. 2013;33:2723-35.

Verma SK, Modi A, Singh AK, Teotia R, Kadam S, Bellare J. Functionally coated polyethersulfone hollow fiber membranes: a substrate for enhanced HepG2/C3A functions. Colloids Surf B Biointerfaces. 2018a;164:358-69.

Verma SK, Modi A, Bellare J. Three-dimensional multiscale fiber matrices: development and characterization for increased HepG2 functional maintenance for bio-artificial liver application. Biomater Sci. 2018b;6(2): $280-91$.

Wang A, Ao Q, Wei Y, Gong K, Liu X, Zhao N, et al. Physical properties and biocompatibility of a porous chitosan-based fiber-reinforced conduit for nerve regeneration. Biotechnol Lett. 2007;29(11):1697-702.

Wang CY, Liu JJ, Fan CY, Mo XM, Ruan HJ, Li FF. The effect of aligned core-shell nanofibres delivering NGF on the promotion of sciatic nerve regeneration. J Biomater Sci Polym Ed. 2012;23(1-4):167-84.

Wang W, Itoh S, Yamamoto N, Okawa A, Nagai A, Yamashita K. Enhancement of nerve regeneration along a chitosan nanofiber mesh tube on which electrically polarized beta-tricalcium phosphate particles are immobilized. Acta Biomater. 2010;6(10):4027-33.

Wei D, Sun J, Yang Y, Wu C, Chen S, Guo Z, et al. Cell alignment guided by nano/micro oriented collagen fibers and the synergistic vascularization for nervous cell functional expression. Materials Today Chemistry. 2018;8:8595. 
Wolfe SP, Hsu E, Reid LM, Macdonald JM. A novel multi-coaxial hollow fiber bioreactor for adherent cell types. Part 1: Hydrodynamic studies. Biotechnol Bioeng. 2002;77(1):8390.

Yang F, Murugan R, Wang S, Ramakrishna S. Electrospinning of nano/micro scale poly(llactic acid) aligned fibers and their potential in neural tissue engineering. Biomaterials. 2005;26(15):2603-10.

Yu X, Bellamkonda RV. Tissue-engineered scaffolds are effective alternatives to autografts for bridging peripheral nerve gaps. Tissue Eng. 2003;9(3):421-30.

Yucel D, Kose GT, Hasirci V. Polyester based nerve guidance conduit design. Biomaterials. 2010;31(7):1596-603.
Zamani F, Amani-Tehran M, Latifi M, Shokrgozar MA, Zaminy A. Promotion of spinal cord axon regeneration by $3 \mathrm{D}$ nanofibrous coresheath scaffolds. J Biomed Mater Res A. 2014; 102(2):506-13.

Zeilinger K, Freyer N, Damm G, Seehofer D, Knöspel F. Cell sources for in vitro human liver cell culture models. Exp Biol Med (Maywood). 2016;241(15):1684-98.

Zeng CG, Xiong Y, Xie G, Dong P, Quan D. Fabrication and evaluation of PLLA multichannel conduits with nanofibrous microstructure for the differentiation of NSCs in vitro. Tissue Eng Part A. 2014;20(5-6):1038-48.
Zhang D, Ni N, Chen J, Yao Q, Shen B, Zhang Y, et al. Electrospun SF/PLCL nanofibrous membrane: a potential scaffold for retinal progenitor cell proliferation and differentiation. Sci Rep. 2015;5:14326.

Zhang HC, Chen TL, Yuan YG. CN patent No. CN 85108751. 1987.

Zhang J, Zhao X, Liang L, Li J, Demirci U, Wang $\mathrm{S}$. A decade of progress in liver regenerative medicine. Biomaterials. 2018;157:161-76.

Zhang N, Zhang C, Wen X. Fabrication of semipermeable hollow fiber membranes with highly aligned texture for nerve guidance. J Biomed Mater Res A. 2005;75(4):941-9.

Zhu Y, Wang A, Patel S, Kurpinski K, Diao E, Bao $\mathrm{X}$, et al. Engineering bi-layer nanofibrous conduits for peripheral nerve regeneration. Tissue Eng Part C Methods. 2011;17:705-15. 